\title{
Structural insight into TNF- $\alpha$ inhibitors through combining Pharmacophore-based virtual screening and Molecular Dynamic Simulation
}

Hina Qaiser ${ }^{\mathrm{a}, \mathrm{b}}$, Maria Saeed ${ }^{\mathrm{a}}$, Dmitry Nerukh ${ }^{\mathrm{b}}$, and Zaheer Ul-Haqa,**

${ }^{a}$ Dr. Panjwani Center for Molecular Medicine and Drug Research, International Center for

Chemical and Biological Sciences, ${ }^{b}$ Department of Mathematics, Aston University, Birmingham

B4 7ET, United Kingdom.

*Corresponding author: Zaheer Ul-Haq, Email: zaheer.qasmi@iccs.edu 


\section{Abstract}

Tumor Necrosis Factor-alpha (TNF- $\alpha$ ), a multifunctional cytokine responsible for providing resistance against infections, inflammation, and cancers. TNF- $\alpha$ has emerged as a promising drug target against several autoimmune and inflammatory disorders. Several synthetic antibodies (Infliximab, Etanercept, and Adalimumab) are available, but their potential to cause severe side effects has prompted them to develop alternative small molecules-based therapies for inhibition of TNF- $\alpha$. In the present study, combined in silico approaches based on pharmacophore modeling, virtual screening, molecular docking, and molecular dynamics studies were employed to understand significant direct interactions between TNF- $\alpha$ protein and small molecule inhibitors. Initially, four different small molecule libraries ( 17.5 million molecules) were virtually screened against the selected pharmacophore model. The identified hits were further subjected to molecular docking studies. The three potent lead compounds (ZINC05848961, ZINC09402309, ZINC04502991) were further subjected to $100 \mathrm{~ns}$ molecular dynamic studies to examine their stability. Our docking and molecular dynamic analysis revealed that the selected lead compounds target the TNF receptor (TNFR) and efficiently block the production of TNF. Moreover, in silico ADMET (Absorption, Distribution, Metabolism, Excretion and Toxicity) analysis revealed that all the predicted compounds have good pharmacokinetic properties with high gastrointestinal absorption and a decent bioavailability score. Furthermore, toxicity profiles further evidenced that these compounds have no risk of being mutagenic, tumorigenic, reproductive and irritant except ZINC11915498. In conclusion, the present study could serve as the starting point to develop new therapeutic regimens to treat various TNF- related diseases. 
Keywords: Autoimmunity; docking; pharmacophore; TNF receptor; MD simulation.

Abbreviations: ADMET; Absorption, Distribution, Metabolism, Excretion and Toxicity; AMBER99; Assisted Model Building with Energy Refinement99; AUC; Area Under Curve; Ar; Aromatic; DUDE; A Database of Useful Decoys Enhanced; EF; Enrichment Factor; Gromacs; Groningen Machine for Chemical Simulations; MD; Molecular Dynamics; MOE; Molecular Operating Environment; PDB; Protein Data Bank; PAINS; Pan-assay interference compounds; TNF- $\alpha$; Tumor Necrosis Factor Alpha. 


\section{Introduction}

TNF- $\alpha$ is an important proinflammatory cytokine responsible for several physiological and pathological conditions including Rheumatoid arthritis (McInnes, Buckley \& Isaacs, 2015), Inflammatory bowel diseases (IBD) (Sticherling, 2016), Ankylosing spondylitis (Maxwell et al., 2015), Psoriasis (Adegbola, Sahnan, Warusavitarne, Hart \& Tozer, 2018) and gastric-carcinoma (Jang et al., 2009). It is chiefly produced by macrophages/ monocytes, mast cells, fibroblast, and natural killer cells during the acute phase reaction. TNF- $\alpha$ is involved in various signaling events inside the cells, leading to necrosis or apoptosis (Idriss \& Naismith, 2000). It exists in two biological forms; membrane-bound TNF (m-TNF) and secreted TNF(s-TNF) released after proteolytic cleavage of the membrane bound iso-form. s-TNF is present in blood plasma and circulate in the body to perform its endocrine function. Both forms of TNF- $\alpha$ (m-TNF \& s-TNF) are biologically active having different functions (Wajant, Pfizenmaier \& Scheurich, 2003, Palladino, Bahjat, Theodorakis \& Moldawer, 2003). The signaling of TNF begins when TNF- $\alpha$ binds to its cognate receptors (TNFRs) such as TNFR1 (Tumor Necrosis Factor Receptor 1) and TNFR2 (Tumor Necrosis Factor Receptor 2) (Liang et al., 2013). TNFR1 is found in many types of cells, while TNFR2 is expressed only on the immune cells. The two receptor types; TNFR1 and TNFR2, tend to bind soluble and membrane-bound forms of TNF $\alpha$, respectively (Chan, Siegel \& Lenardo, 2000, Sedger \& McDermott, 2014).

TNF inhibition has been achieved by blocking TNF binding to its receptors to avoid the adverse effects (Papadakis \& Targan, 2000). The pleiotropy of TNF validates it as a therapeutic target for the treatment of various autoimmune and inflammatory disorders. Some FDA approved synthetic biologics (antibodies) including Infliximab, Adalimumab, Etanercept, Certolizumab and 
Golimumab (Bongartz, Matteson \& Orenstein, 2005, Jacobi, Mahler, Schuler \& Hertl, 2006, Monaco, Nanchahal, Taylor \& Feldmann, 2014) inhibits TNF- $\alpha$ directly and successfully used for the treatment of some inflammatory diseases including Crohn's disease, Rheumatoid arthritis and Ulcerative colitis (Mesaik et al., 2012). However, these therapeutic biologics have several side effects including the weakening of body defensive system by eliciting antigen-antibody response against self-antigens, high cost and painful administration (Hochberg, Lebwohl, Plevy, Hobbs \& Yocum, 2005, Sfikakis \& Tsokos, 2011, Lis, Kuzawińska \& Bałkowiec-Iskra, 2014). The dilemma has made researchers pay more attention towards designing small molecule inhibitors against TNF- $\alpha$. Currently, small molecule-based therapies are the most promising alternative approach to develop potent TNF- $\alpha$ inhibitors because they are low cost, easy to manufacture and deliver to the target tissue (Davis \& Colangelo, 2013). Unfortunately, very few small-molecule inhibitors are available, but none of them has reached clinical trials. It is very challenging to find a low molecular weight compound that interacts directly with protein to block its function (Buchwald, 2010). To date, most small molecules interact with TNF- $\alpha$ indirectly by downregulating its expression and some inhibitors promote direct disassembly of TNF- $\alpha$ with its receptors (Berg, 2003). Suramin and SPD307 promote disassembly of TNF with its receptor by disrupting the binding of TNF- $\alpha$ trimer complex but not considered as potent TNF inhibitors due to their low potency and toxicity (Mancini et al., 1999, He, 2005).

Even after such extensive work, due to lower potency and higher cytotoxicity profiles of small molecular inhibitors researchers still target TNF- $\alpha$ to find more potent inhibitors to reduce the higher cost and side effects of currently available treatments. Therefore, in the present study, we used ligand-based pharmacophore modeling, virtual screening, molecular docking studies to identify the novel TNF- $\alpha$ inhibitors. In this connection, we further performed the MD simulation 
of selected hits to gain insight into the real dynamic behavior and binding mechanism of TNFalpha with its inhibitors. Moreover, pharmacokinetic and drug-likeness properties of selected hits were also determined to evaluate their potential to act as a drug candidate for TNF- $\alpha$.

\section{Material and methods}

\subsection{Data set}

A set of 27 TNF- $\alpha$ inhibitors was collected from literature based on their experimental inhibitory activity (IC 50 values) ranging from $0.004 \mu \mathrm{M}$ to $60 \mu \mathrm{M}$ (Fujita, 2002, Cheng \& et al., 2004, Laufersweiler \& et al., 2004, Sabat \& et al., 2006, Dhuru et al., 2011, Mouchlis, et al., 2012, Shah et al., 2012, Guirado et al., 2013, Kim et al., 2014). The 3D structure of these compounds was sketched by using ChemDraw Ultra 11.0 software (Li et al., 2004) followed by atom typing correction and geometry optimization by Sybyl Tripos 7.3. (SYBYL7.3, 2007). Energy minimization of the dataset was performed by the Tripos molecular mechanics force field through Powell's conjugate gradient method (Clark, Cramer, and Van Opdenbosch, 1989) with one thousand steps and $0.5 \mathrm{kcal} / \mathrm{mol} \AA$ A threshold. To calculate partial atomic charges Gasteiger-Huckel charges (Gasteiger, 2005) were applied to all the ligands by AutoDock 4.2 software (Morris et al., 2009) and hydrogen atoms were added to the whole dataset. These 27 reported inhibitors belong to the diverse classes of inhibitors and they were used for the ligand-based pharmacophore generation. The 2D structures and inhibitory activity of these compounds are present in Table $\mathbf{1}$. 
Table 1: List of reported active compounds and their corresponding $\mathrm{IC}_{50}$ values in $\mu \mathrm{M}$ collected from the literature.

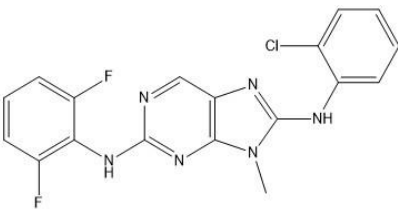

1. $1.5 \mu \mathrm{M}$

(Sabat \& et al., 2006)

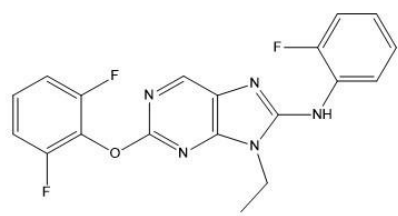

4. $0.035 \mu \mathrm{m}$

(Sabat \& et al.,2006)

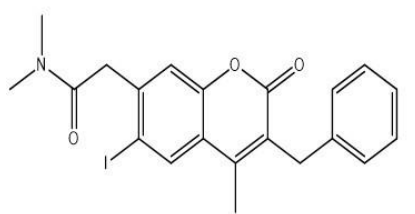

7. $0.06 \mu \mathrm{M}$

(Cheng \& et al., 2004)

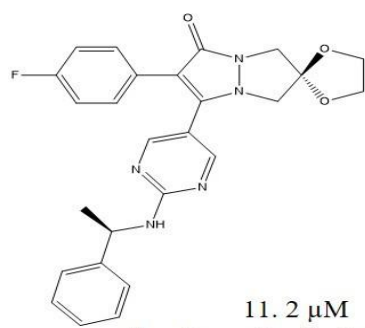

(Laufersweiler \& et al., 2004)

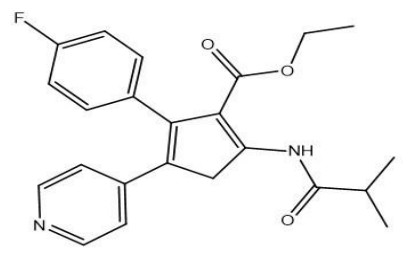

14. $36 \mu \mathrm{M}$ (Fujita, 2002)

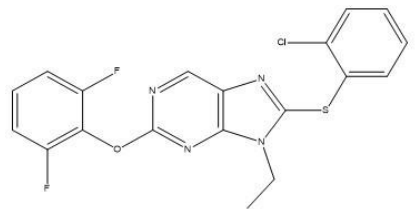

2. $0.014 \mu \mathrm{m}$

(Sabat \& et al., 2006)

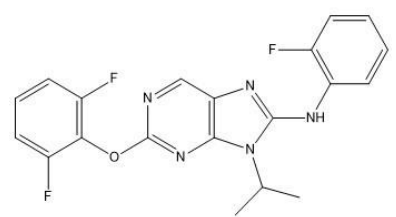

5. $0.004 \mu \mathrm{m}$ (Sabat \& et al.,2006)<smiles>Cc1cc2c(C)c(Cc3ccccc3)c(=O)oc2cc1CC(=O)C(C)C</smiles>

8. $0.06 \mu \mathrm{M}$ (Cheng \& et al., 2004)

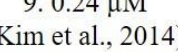

3. $0.048 \mu \mathrm{m}$

(Sabat \& et al.,2006)

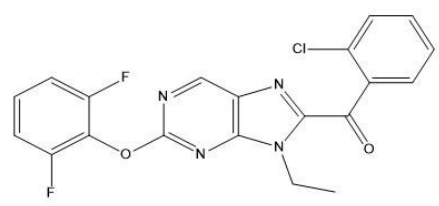

6. $0.004 \mu \mathrm{m}$

(Sabat \& et

al.,2006)

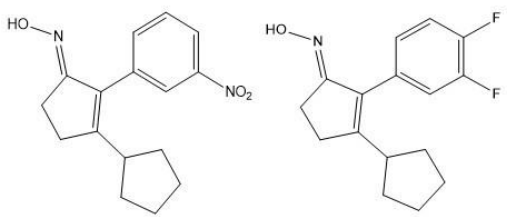

10. $0.26 \mu \mathrm{M}$

(Kim et al., 2014)

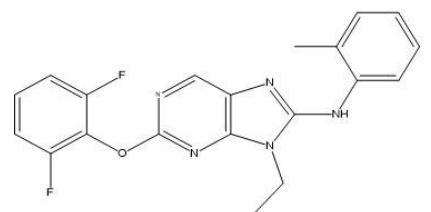

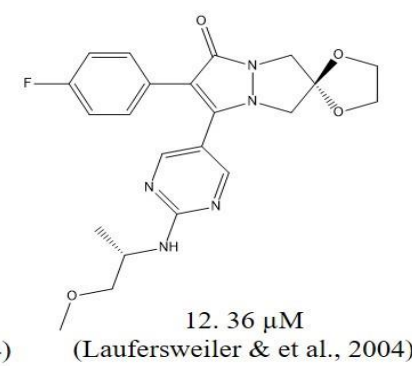

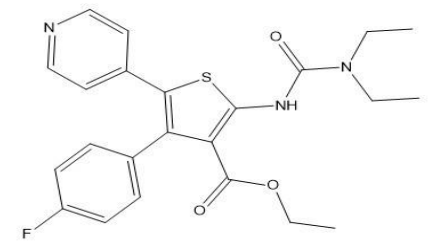

15. $2.6 \mu \mathrm{M}$ (Fujita, 2002)

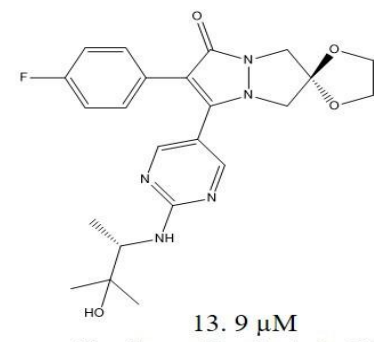

(Laufersweiler \& et al., 2004)

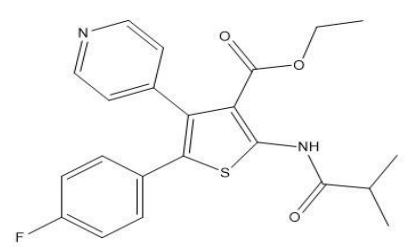

16. $5.4 \mu \mathrm{M}$ (Fujita, 2002) 


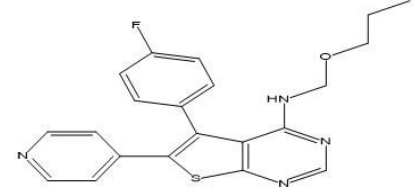

17. $10 \mu \mathrm{M}$

(Fujita, 2002)

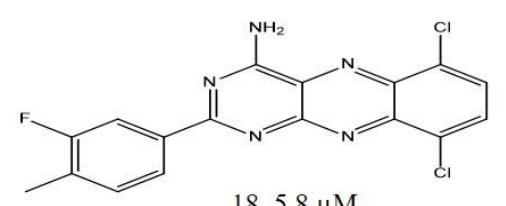

18. $5.8 \mu \mathrm{M}$

(Guirado et al., 2013)

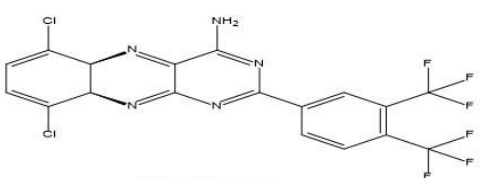

19. $7.3 \mu \mathrm{M}$

(Guirado et al., 2013)
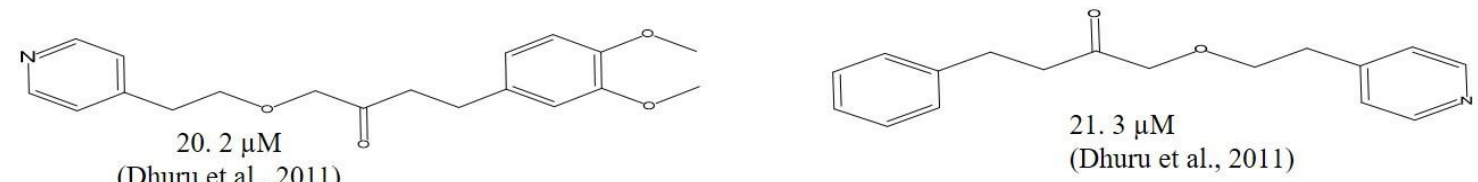

(Dhuru et al., 2011)

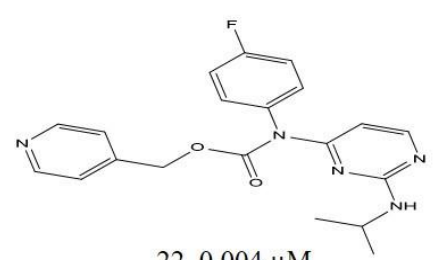

22. $0.004 \mu \mathrm{M}$

(Mouchlis, et al., 2012),

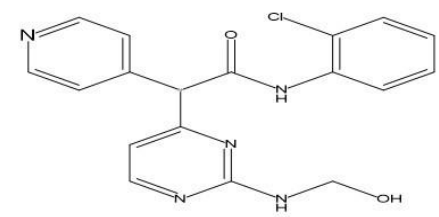

25. $0.071 \mu \mathrm{M}$

(Mouchlis, et al., 2012)

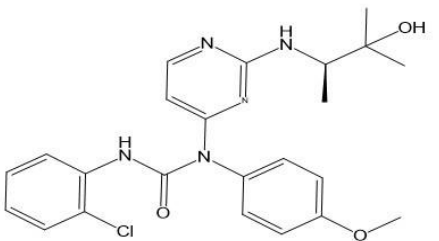

23. $0.092 \mu \mathrm{M}$

(Mouchlis, et al., 2012)

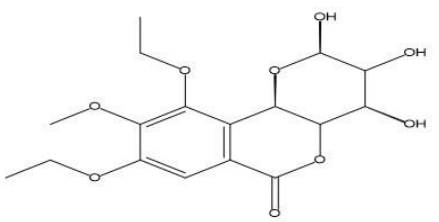

26. $31 \mu \mathrm{M}$

(Shah et al., 2012)

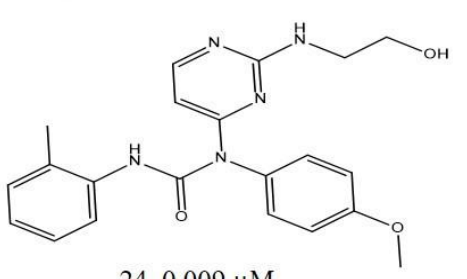

24. $0.009 \mu \mathrm{M}$

(Mouchlis, et al., 2012)

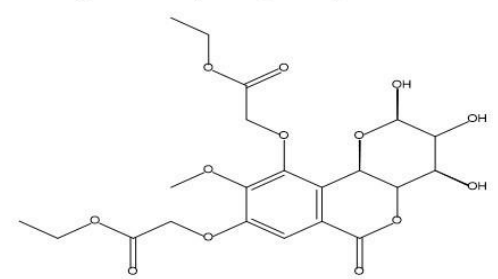

27. $60 \mu \mathrm{M}$

(Shah et al., 2012)

\subsection{Receptor preparation}

To get initial coordinates of $\mathrm{TNF}-\alpha$, the crystal structure of human tumor necrosis factor-alpha with reported inhibitor (307) measured at 2.1 resolution was downloaded from RCSB Protein Data Bank under the accession code 2AZ5 (Berman, 2000, He, 2005). TNF- $\alpha$ is a homotrimer, containing three chains $(\mathrm{A}, \mathrm{B}, \mathrm{C})$ but chain $\mathrm{C}$ was removed due to its similarity with chains $\mathrm{A}$ and B (Tang, Hung \& Klostergaard, 1996). Therefore, chains A and B were selected because the binding pocket of TNF- $\alpha$ lies at the interface of these two chains (He, 2005). Protein preparation 
started from the modeling of missing residues via Modeller9.23 software (Šali \& Blundell, 1993) and the quality of model was assessed using PROCHECK (Laskowski, MacArthur, Moss \& Thornton, 1993), ERRAT (Colovos \& Yeates, 1993) and Verify3D (Bowie, Luthy \& Eisenberg, 1991, Lüthy, Bowie \& Eisenberg, 1992) online servers, followed the addition of missing bonds and atoms, removal of heteroatoms, and water molecules using the protein preparation module in MOE2019.01 software (Vilar et al., 2008). Further Hydrogen atomes were added by using the protonate 3D algorithm, while partial charges were assigned via AMBER99 Force Field implemented in MOE2019.01.

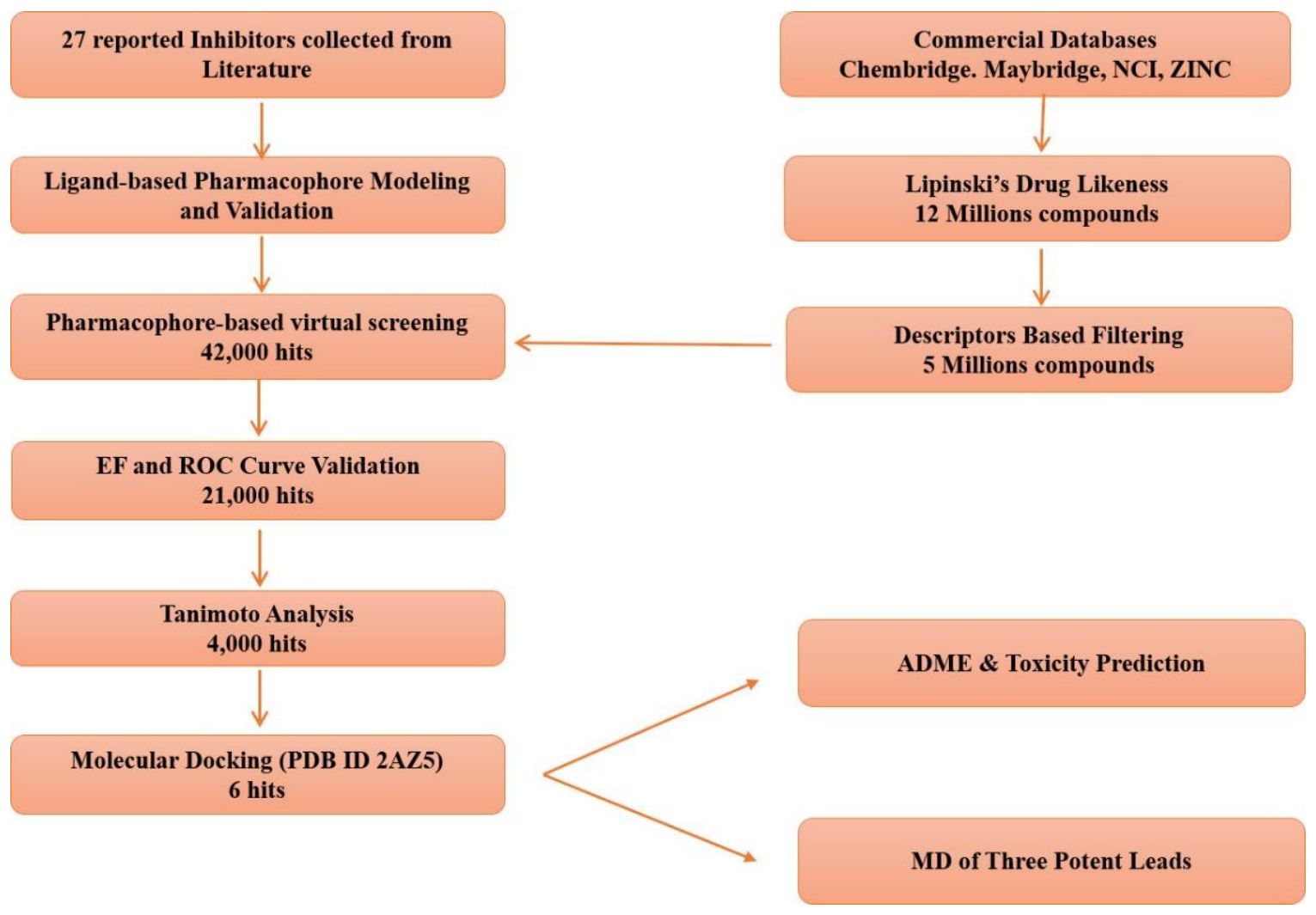

Figure 1. represents the graphical summary of the workflow. 


\subsection{Ligand-Based pharmacophore modelling}

In our study, a single crystallized complex structure (PDB ID: 2AZ5) was available. As a compensation, we employed the coordinates of the ligand protein complexes, obtained after docking the reported inhibitors with PDB: 2AZ5, to generate structure-based pharmacophore models. However, the models failed to produce significant features for pharmacophore generation. Therefore, ligand-based pharmacophore modeling has been implemented in this study. All the ligand-based pharmacophore modeling studies were performed using Ligandscout4.2 (Wolber \& Langer, 2005). The ligand-based approach requires the active conformation of reported inhibitors to develop a model. In this study, information extracted from 27 reported TNF inhibitors was used to create pharmacophores, which were used in different combinations for modeling pharmacophores with either merged or shared features, or combination of both. The generated models were further with the help of active, decoys and random datasets of compounds.

\subsection{Validation of Generated Pharmacophore Model}

The developed pharmacophores were analyzed to validate their ability to extract active molecules from a large data set of unknown molecules. In the present study, three datasets such as actives, random, and decoys were used for the validation of constructed pharmacophore model. The test set of active compounds contained 27 reported TNF- $\alpha$ inhibitors retrieved from the literature based on their good inhibitory activity $(0.004 \mu \mathrm{M}$ to $0.323 \mu \mathrm{M})$ (Table 1). The random dataset comprised 80 compounds (Table S2) that were collected randomly from various commercial databases and have not been tested against TNF protein. The third dataset was created by using DUDE (Directory of useful decoys enhanced) web server (Mysinger, Carchia, Irwin \& Shoichet, 2012). The DUDE server use the information of known active compounds to generate the unique dataset for the 
validation of our generated pharmacophore model. Twenty-seven active compounds were submitted in smile format to this web server that generated 50 decoys for every compound, hence it provided us with a total of 1350 compounds that have similar physical and chemical properties to the active compounds illustrated in (Table S3). All the datasets (test, decoys, and random) were converted from mol2 to ldb (ligands out database) format with the help of the idbgen module of LigandScout4.2 software. These datasets were then ready to use for the validation process of our generated pharmacophore models. This validation protocol is important because it ensures that our generated model is reliable, and it can detect the known active molecules correctly from the large data of unknown molecules.

\subsection{Virtual screening}

The main goal of screening the virtual database is to find those potential lead compounds which slightly vary in their chemical structure but with strong inhibitory activity against the target receptor. All the dataset of screening compounds was downloaded from 4 commercially available compounds libraries including Chembridge (Eugene et al., 2010) ( 50,000 compounds), Maybridge (ScrGuide, 2004) ( 10,6,478 compounds), NCI (Stinson et al., 1992) ( 2,65,242compounds) and ZINC (Irwin and Shoichet, 2005) ( 17,000000 compounds). Initially, 17.5 million compounds from all the four databases were checked for their drug-likeness properties by using the Lipinski rules (Lipinski, 2004). Moreover, obtained hits were passed through the second filter based on their similarity (2D features) with the reported TNF- $\alpha$ inhibitors (Table S1). Furthermore, these compounds were subjected to the third filter by using the Tanimoto coefficient (Willett, Barnard \& Downs, 1998) analysis. In Tanimoto coefficient analysis many conformations have been generated against query molecule then overlapping of both molecules 
takes place and it assigns the similarity index (ranges 0-1) to the test compound which shows similarity with the query compound. After creating a virtual database, it has been converted into the Tripos multimol2 files and subjected to the minimization step to achieved most stable and lowest energy conformation by using omega module of OpenEye software (OpenEye scientific software), then gasteiger charges were applied, and hydrogens were added to all compounds. Finally, the ligand dataset is prepared for the assessment of the docking procedure against the selected TNF protein.

\subsection{Statistical Analysis (Enrichment factor (EF) \& ROC curve)}

The virtual screening results were evaluated by their enrichment factor (Bender \& Glen, 2005) and Receiver operating curve analysis (Truchon \& Bayly, 2007, Triballeau, Acher, Brabet, Pin \& Bertrand, 2005). Enrichment factor is defined as the concentration of active compounds in a set of virtual screening results for assessment. It can be calculated by the following formula:

$$
\text { Enrichment Factor }=\underline{\text { Actives (in subset) } / \text { Actives (in total dataset) }}
$$

No. of compounds( subset) / No. of compounds (in the total dataset)

ROC curve is another powerful method that provides accuracy of virtual screening result's and it has the potency to differentiate actives from the in-actives and create a boundary between them (Empereur-mot et al., 2015). It is created by plotting the true positive rate against the false-positive rate at several threshold settings. The area under the curve (AUC) is used to access the performance of the overall method and its values range from 0 to 1 . 


\subsection{Molecular Docking}

Molecular docking simulation was performed using Auto Dock 4.2 (Morris et al., 2009) software due to its reproducible results in our redocking experiment (RMSD 1.3 $⿱$ A). It is an automated docking software including a rigid receptor along with its flexible side chains and ligands that results in the best ligand binding pose with minimal binding energy (Morris et al., 2009). Auto Dock needs a pre-calculated grid map of each ligand atom and this grid must surround the active site of the protein. Koll man's charges (Singh \& Kollman, 1984) were applied to protein and Gasteiger charges (Gasteiger, 2005) were applied to the ligands due to their non-peptide nature. Auto grid 4.2 (Morris et al., 2009) was used to create a grid box of $38 \AA$ x $36 \AA$ x $38 \AA$ around the protein, it provides a rigid body cavity during docking simulations. The Lamarckian genetic algorithm (Fuhrmann, Rurainski, Lenhof \& Neumann, 2010) was used to search for the best-fit ligand conformers. The grid spacing of $0.375 \AA$ was suitable for docking and 1000000 times energy evaluation was performed for every ligand. Ten conformers were generated for every ligand and best-ranked ligand binding poses were selected for further evaluation.

\section{$2.8 \quad$ Re-docking}

Re-docking experiments were performed to check the software's ability to reproduce the crystal pose of the cognate ligand. For this purpose, the performance of five software packages (Auto Dock4.2, MOE2019.1, GOLD, FRED and Surflex Dock.) were evaluated based on root mean square deviation (RMSD). RMSD serves as a gold standard to evaluate the reliability of the docking protocol (Ajmal Shah, Khalil, Ul-Haq \& Panichayupakaranant, 2017). For a reliable redocking experiment, RMSD value between the cartesian coordinate of redock and original 
crystal binding pose must be less than $3 \AA$. The ligand and receptor were prepared for re-docking experiments according to the software's requirement.

\subsection{Molecular Dynamic (MD) Simulation}

To explore the structural and dynamic behavior in protein-ligand complexes 100 nano-second MD simulation was performed by utilizing the top three virtual hits and reference compounds as initial structures. Gromacs 5.1.4 software package (Abraham et al., 2015) was used to performed MD simulation. pdb2gmx tool was used to generate the coordinate topological file for target protein using the "GROMOS96 54a7" force field (Scott et al., 1999). The ligands topological files were generated by the ATB server (Malde et al., 2011) it converts coordinates of small molecules into pdb format. Each system was solvated in a cubic box with the protein placed at $10 \AA$ distance from the box wall and SPC216 (Mark \& Nilsson, 2001) (simple point charge) solvent model was used within the periodic boundary condition (PBC). All the systems were neutralized by the addition of counterions followed by the energy minimization of all the complexes in 50,000 steps to get rid of steric clashes. After the minimization step, all the systems were equilibrated to $300 \mathrm{~K}$ temperature for 100ps under NVT condition (constant temperature and volume) which further followed by NPT i.e. (temperature $300 \mathrm{~K}$ and 1.0 atmospheric pressure) ensemble for 100ps along with periodic boundary condition. The temperature and pressure were coupled to the Berendsen thermostat and barostat (Berendsen, Postma, van Gunsteren, DiNola \& Haak, 1984) with a coupling time of 0.1 ps. The LINCS algorithm (Hess, Bekker, Berendsen \& Fraaije, 1997) was used to fix the bond lengths and Coulomb and Vander Waals interactions were treated with a single cut off $10 \AA$. Particle Mesh Ewald (PME) (Darden, York \& Pedersen, 1993, Essmann et al., 1995) was used for treating long-range electrostatic interaction. Finally, production MD run of 100ns was carried out 
and snaps shots were generated after every 2 femtoseconds to observe the real-time dynamics and stability of each complex. The results of MD Simulations were examined by using different modules of Gromacs software and graphs were plotted via the XMGrace tool.

\subsection{In silico Absorption, Distribution, Metabolism, Excretion and Toxicity (ADMET) prediction}

To predict the pharmacokinetics and drug-likeness properties of selected hits compounds were submitted in the canonical smiles format to the SwissADME online server (Daina, Michielin \& Zoete, 2017). This analysis was performed to predict the pharmacokinetic properties of hits such as Gastrointestinal absorption, P-glycoprotein ( $\mathrm{p}-\mathrm{gp})$, Blood-brain Barrier permeability and inhibition of Cytochrome P450 isoforms. Moreover, drug-likeness and bioavailability score prediction was also conducted according to Lipinski (Lipinski, Lombardo, Dominy \& Feeney, 2001), Ghose (Ghose, Viswanadhan \& Wendoloski, 1999), Veber (Veber et al., 2002), Egan (Egan, Merz, \& Baldwin, 2000), Muegee (Muegge, 2003) rules. The drug-likeness rules by Lipinski, Ghose, and Weber were applied to predict drug-likeness of the molecules based on some important parameters such as MW (Molecular Weight), Slogp, $\log S$ and number of HBD and HBA. Egan rules provide a prediction of drug absorption properties based on the membrane permeability, while Muegee rules provide a prediction of pharmacophore points that differentiate between drug-like and non-drug-like molecules. The SwissADME online server utilizes a support vector machine algorithm (Cortes \& Vapnik, 1995) with large datasets of known and noninhibitors along with substrates and non-substrates. The Toxicity profiles of hits compounds were analyzed by using Osiris DatawarriorV.5.0.0 (Sander, Freyss, von Korff \& Rufener, 2015). 


\section{Results and discussion}

Successful hits to lead optimization campaigns discovered few potent TNF- $\alpha$ inhibitors. For example, Chan et al identified two potent molecules (quinuclidine 1 and indoloquinolizidine 2) by ligand-based virtual screening and their experimental findings suggested that quinuclidine 1 has better efficacy than indoloquinolizidine 2 with the inhibitory concentration of $5 \mu \mathrm{M}$ and more compared to $30 \mu \mathrm{M}$, respectively (Chan et al., 2010). Chio et al identified a potent compound (Oxole-1) using structure-based virtual screening that showed good inhibition of TNF at $10 \mu \mathrm{m}$. (Choi, Lee, Park \& Oh, 2010). A novel metal-based TNF inhibitor was also reported by Leung et al (Leung et al., 2012). Mouhsine et al. used combined in silico and in vitro screening methods to discover orally available TNF inhibitors (Mouhsine et al., 2017). Liang et. al, performed screening of bi-cyclic peptides libraries for protein-protein interaction inhibitors which lead to the discovery of TNF- $\alpha$ antagonist (Liang et al., 2013). Other works were also reported to discover TNF- $\alpha$ inhibitors. (Leung et al., 2011; Hanumanthappa et al, 2012; Mouchlis et al, 2012; Ma et al., 2014; Alexiou et al., 2014; Kang et al., 2016). Despite these efforts, the search for TNF-inhibitors is taking ground in drug discovery campaigns. For this reason, our ligand-based virtual screening campaign was designed to explore the vital interactions between TNF and its receptors that further used to develop novel and potent TNF-inhibitors.

\subsection{Protein Modelling}

Modeling of missing residues of TNF- $\alpha$ protein was performed via Modeller9.23 software (Šali \& Blundell, 1993) (Figure 2). Some residues were missing in the crystallized structure of TNF- $\alpha$ 
(PDB ID: 2AZ5) as in chain A, 16 residues were missing: Arg31, Arg32, Ala33, Asp34, Ala35, Gln102, Arg103, Glu104, Thr105, Pro106, Glu107, Gly108, Ala109, Glu110, Ala111, and Lys112. In chain B, 8 residues were missing Glu104, Thr105, Pro106, Glu107, Gly108, Ala109, Glu110, and Ala111. After modeling the residues PROCHECK was run to check the stereochemical quality of the modelled protein structure and Ramachandran plot (Ramachandran, Ramakrishnan \& Sasisekharan, 1963) was obtained. Ramachandran plot provides information on torsional or dihedral angles (phi and psi) of amino acid residues in protein structure. It consists of allowed and disallowed regions. The allowed region is low energy regions that are sterically favorable while the disallowed region is sterically unfavorable regions. The Ramachandran plot of our modeled structure shows $98.9 \%$ of residues were lying in favorable and allowed region only $1.2 \%$ ( 3 residues) in the disallowed region indicating the reliability of the modeled structure as depicted in Figure 3.
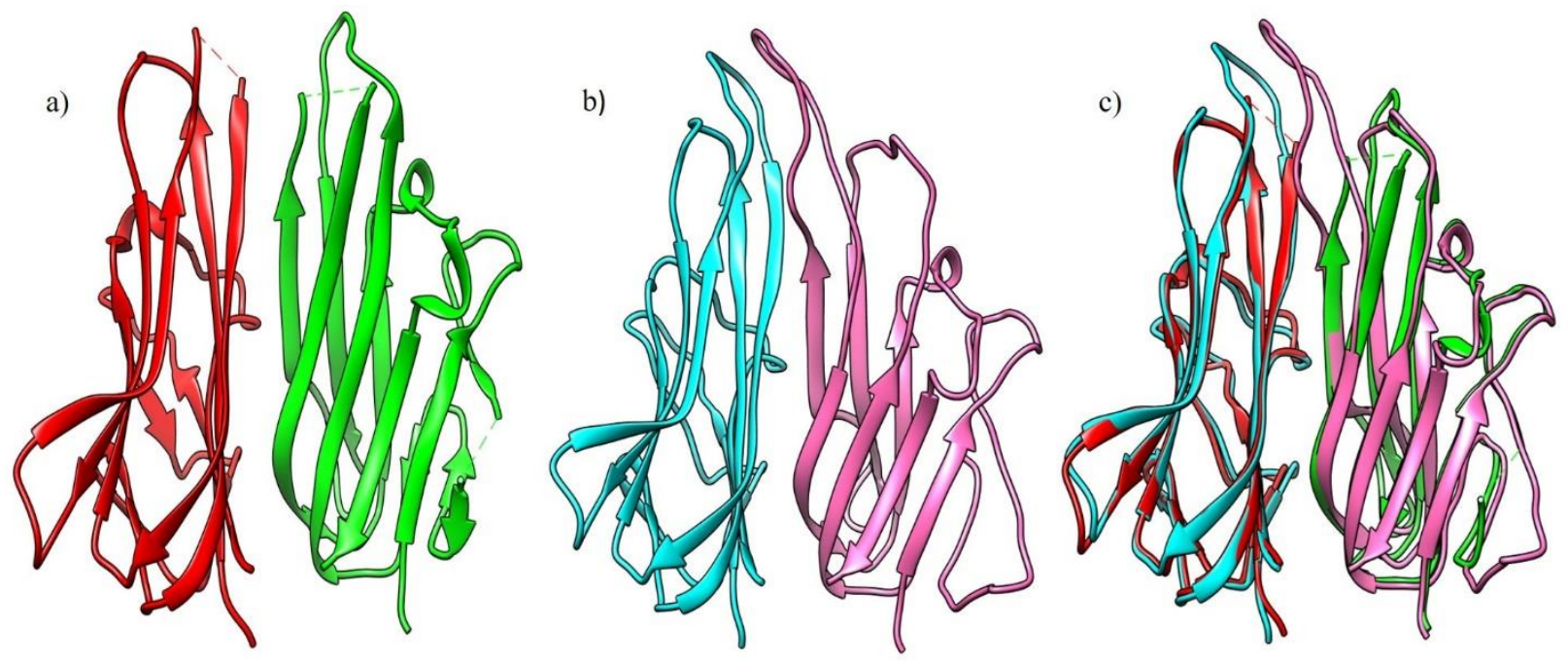

Figure 2. a) Unmodeled Structure of TNF protein. b) Modeled Structure of TNF protein. c) Alignment of modeled and unmodeled TNF protein structure. 


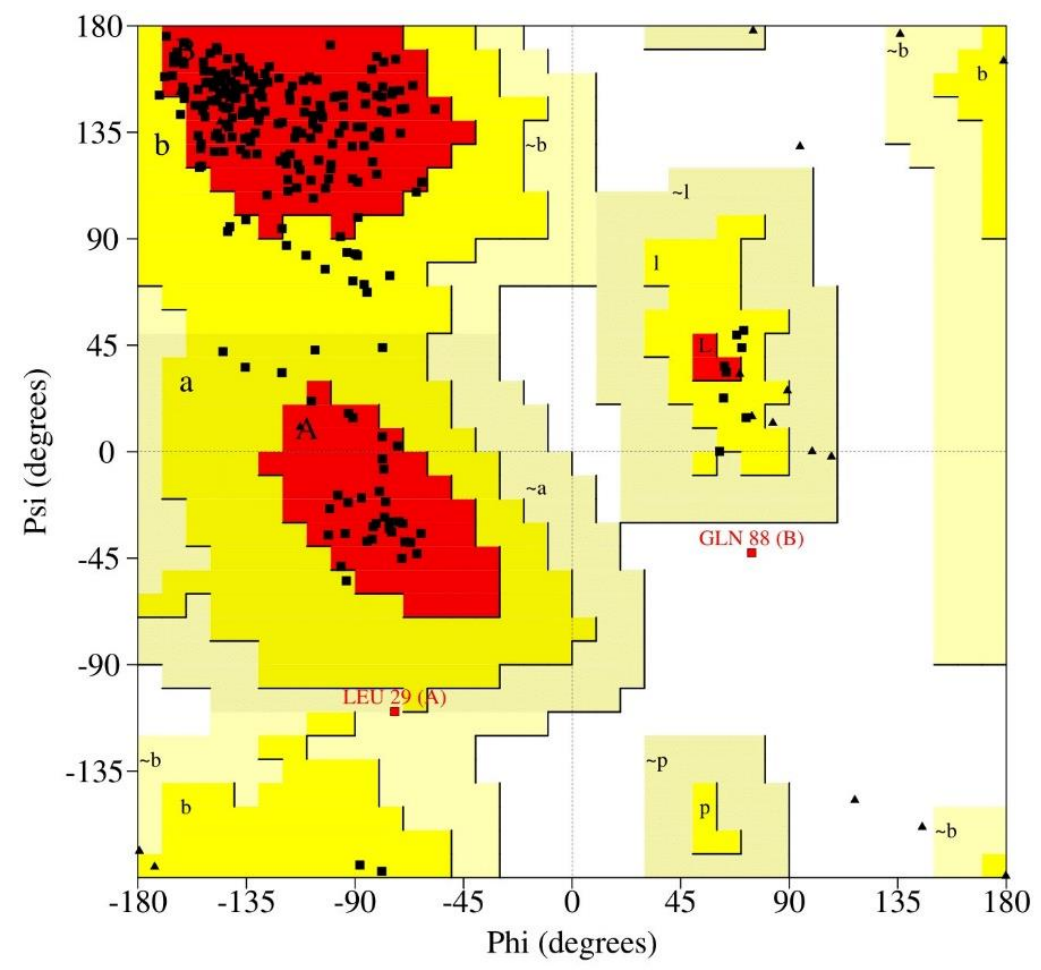

Figure 3. Ramachandran plot of modeled TNF- $\alpha$ protein showing the correlation of phi $(\Phi)$ and psi $(\Psi)$ angles. The most favorable regions are shown in red color, additionally allowed, generously allowed and disallowed regions are shown in brown, yellow and light-yellow color respectively.

\subsubsection{ERRAT model validation}

ERRAT is an online server used to examine non-bonding interactions between atom pairs in the modelled protein's structure. The overall ERRAT quality factor score was 85.606 depicted in Figure 4 indicating good quality of constructed model. 
Program: ERRAT2

File: /home/saves/Jobs/6446964/qq_aaaa.pdb_errat.logf

Overall quality factor**: 85.606

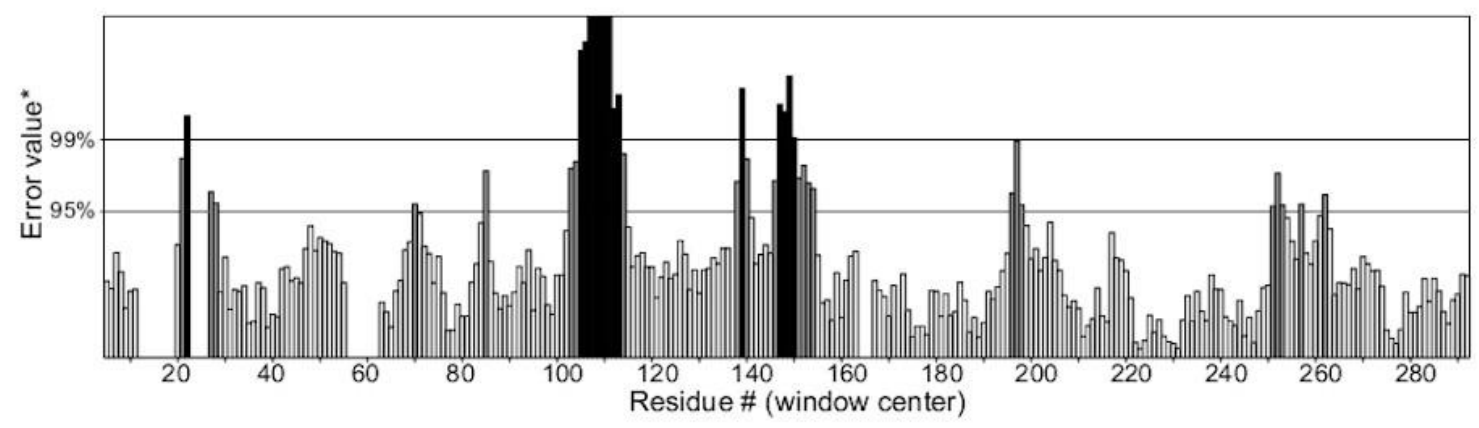

Figure 4. Validation of modelled protein structure via ERRAT tool showing good ERRAT quality factor score.

\subsubsection{Verify3D model validation}

Verify3D analyzes protein structures based on their three-dimensional profiles. It examines the correlation of protein models (3D) and sequences of amino acids in 1-dimensional manner and assigns different structural classes to every residue according to their local environment. The Verify3D plot of our modelled structure shows that $80.7 \%$ residues have scored $>=0.2$ in the 3D/1D profile suggesting the reliability of the constructed model (Figure 5). 


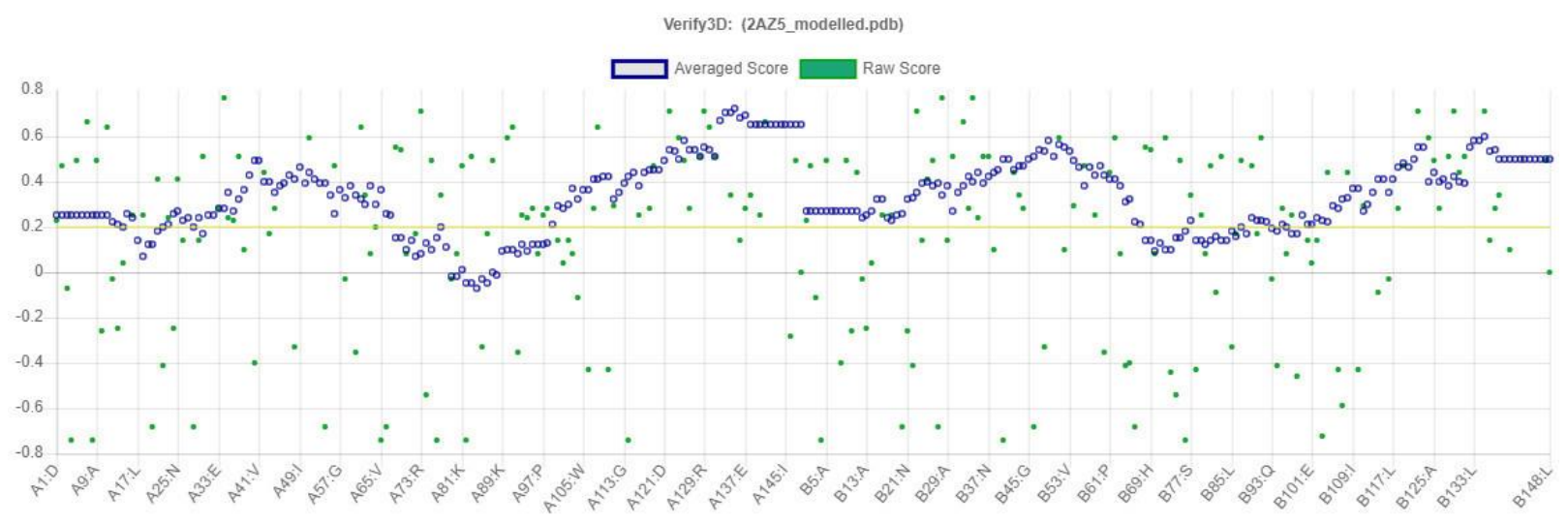

Figure 5. Verify3D analysis of Modelled protein structure showing average 3D-1D score of all residues $>=0.2$ which is acceptable for modelled protein structure.

\subsection{Ligand-based Pharmacophore Generation}

Pharmacophore is defined as a molecular architecture that contains important features necessary for the biological activity of the drug (Gao, Yang \& Zhu, 2010). In the current study, ligand-based pharmacophore modeling was used to generate a reliable pharmacophore model using LigandScout 4.2. For this purpose, 27 experimentally reported compounds that had good inhibitory activity against TNF- $\alpha$ were used to generate several shared or merge ligand-based pharmacophoric models. These generated pharmacophore models were used for virtual screening which leads to the identification of novel and potent lead compounds. In order to generate the model, ligands were aligned, and common features were extracted to find out the good pharmacophore model. Therefore, twelve pharmacophores models were generated by the alignment of three different pharmacophores with various combinations of shared and merge 
features from experimentally reported inhibitors. The selection of these compounds based on their potency and structural diversity. The selected pharmacophore is derived from three potent inhibitors such as Compound 5 (IC50 $0.004 \mu \mathrm{M}$ ), Compound 6 ( $\mathrm{IC}_{50} 0.004 \mu \mathrm{M}$ ) and compound 11 (IC50 $2 \mu \mathrm{M}$ ). Compounds 5 and 11 were presented with sixteen pharmacophoric features including four aromatic rings, six HBA (hydrogen bond acceptors), six hydrophobic and one HBD (hydrogen bond donor). Similarly, compounds 6 contained thirteen pharmacophoric features such as six HBA, four hydrophobic and three aromatic ring features as shown in Figure 6. Six HBA, three hydrophobic and three aromatic rings are found to be common in all three ligand-based pharmacophore models generated by these ligands. However, one HBD feature is restricted only for compounds 5 and 11.
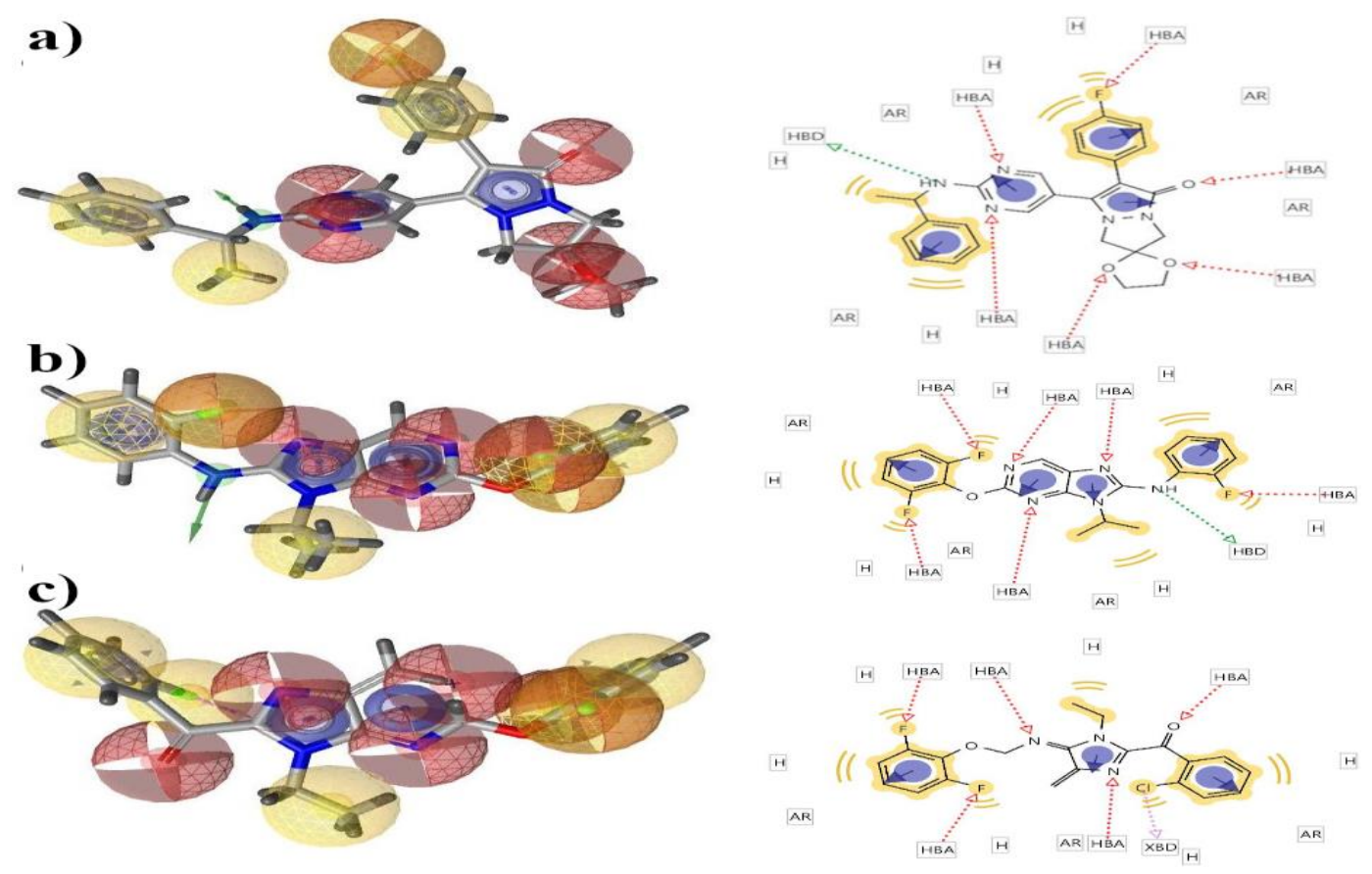

Figure 6. Pharmacophore representations of compound 11 (a) compound 5 (b) and Compound 6 (c) Pharmacophore models generated by LigandScout4.2. Green and red arrows represent H-bond donors and H-bond acceptors and Yellow spheres represent hydrophobic, Blue sphere represents aromatic ring feature respectively. 
Table 2 summarizes the generated pharmacophore models with their statistical parameters. All the generated models comprise of either four or five pharmacophore features (HBA, HBD, Hyd, Ar); all the models were varying in their composition, orientation, and vector directions. The combination of compounds used for the construction of pharmacophore models are shown in Table 2 and here we discussed only the selected pharmacophore model. Model 7 considered as the best pharmacophore model consists of two HBA, one hydrophobic and one aromatic feature as shown in Figure 7. These features were common among all the three active compounds and were necessary for inhibiting TNF- $\alpha$ at the nanomolar level. All the generated pharmacophore models were ranked based on the pharmacophore fit score, a default scoring function implemented in LigandScout software. This scoring function measures the best geometric fit of the features of a compound to the three-dimensional ligand-based pharmacophore model. A high pharmacophore fit score indicates the best fit for the model and those compounds that nicely fit the pharmacophore model should also show good activity at TNF- $\alpha$. In our case, the pharmacophore fit score of all the virtual hits was higher (48.08-46.25) indicating that their chemical feature best aligned to the features of selected pharmacophore model 7 as depicted in Figure 7. 

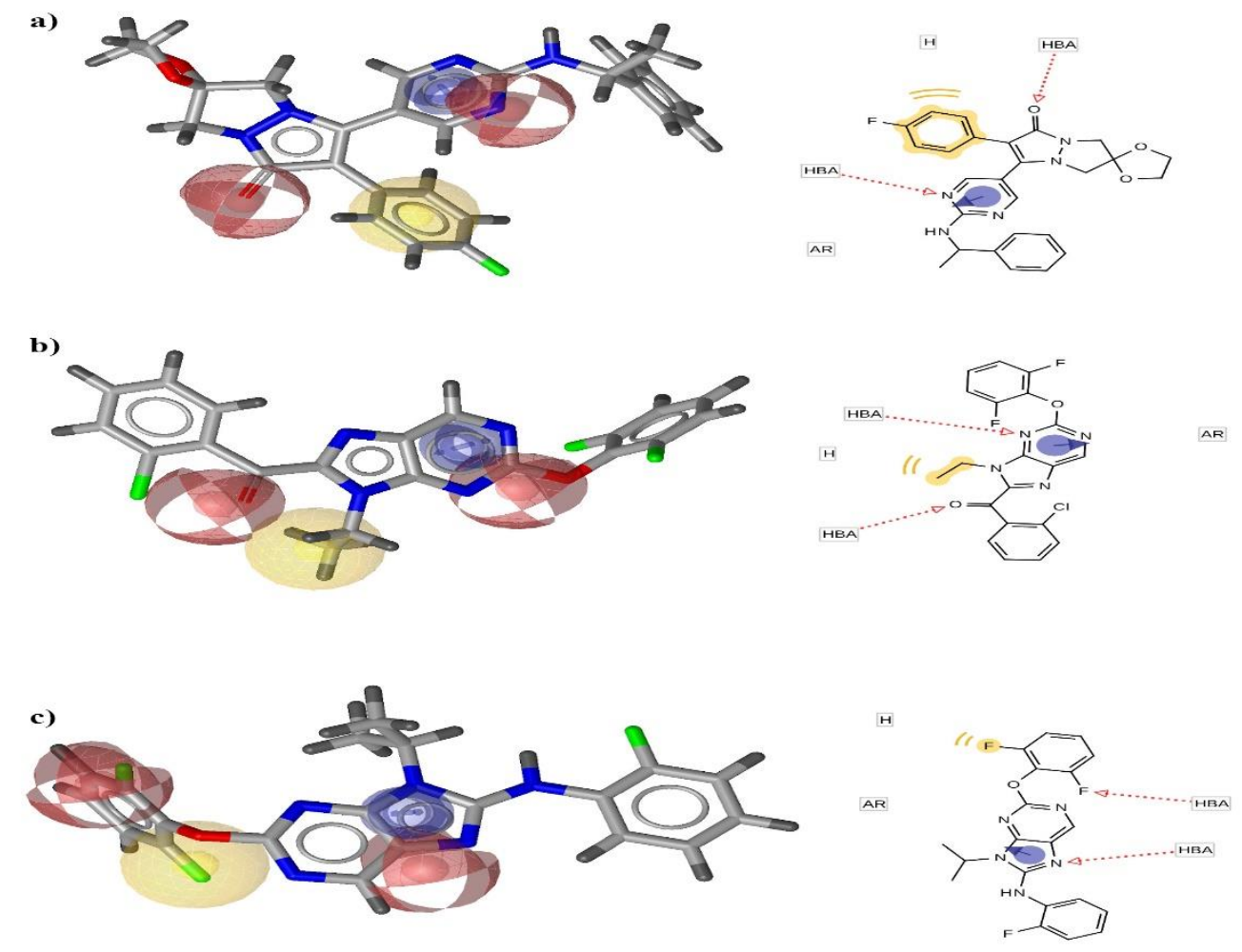

Figure 7. Alignment of selected pharmacophore model 7 with compound 11 (a) compound 5 (b) and Compound 6 (c) Pharmacophore models generated by LigandScout4.1. Green and red arrows represent H-bond donors and Hbond acceptors and Yellow spheres represent hydrophobic areas; Blue sphere represents aromatic ring feature respectively.

\subsection{Validation of Generated Pharmacophore Model}

Prediction of active, inactive, random and decoys data set, was the initial step in the validation of the constructed pharmacophore model. The developed pharmacophore models were utilized for the estimation of hit rates of test datasets. Model 7 showed good hit rates other than developed models. The best pharmacophore model and its hits rates against active, inactive, random and decoys compounds summarize in Table 2. The selected model consisted of one hydrophobic, one 
aromatic and two HBA features. The selected pharmacophore showed $80 \%$ active identifying hit rate. In general, it predicted 22 highly active compounds out of 27 correctly indicating that this pharmacophore could pick compounds with high experimental activity against TNF- $\alpha$. Moreover, the best pharmacophore model was further evaluated by decoys, inactive and random datasets. 9 $\%$ decoys (125 out of 1350), 25\% inactive ( 1 out of 4 ) and $2 \%$ (2 from 80 compounds) random compounds were picked by the selected model 7 showing its good predictive ability to discriminate between active, inactive and decoys dataset. The pharmacophore models having a low picking rate of active compounds were not discussed here. The pharmacophore model (1, 3, 5 and 9) also showed higher picking rate of actives but they also picked decoys and inactive compounds in higher percentages and they cannot be considered as a good pharmacophoric model.

Table 2. Generated Pharmacophore Models (Shared Feature) and their validation results.

\begin{tabular}{|c|c|c|c|c|c|c|c|}
\hline S.no & $\begin{array}{c}\text { Combination } \\
\text { s of } \\
\text { Compounds }\end{array}$ & $\begin{array}{c}\text { Pharmaco } \\
\text { p-hore } \\
\text { model }\end{array}$ & $\begin{array}{l}\text { Shared Features of } \\
\text { Pharmacophores }\end{array}$ & $\begin{array}{c}\text { Actives } \\
(\%)\end{array}$ & $\begin{array}{c}\text { Inactive } \\
(\%)\end{array}$ & $\begin{array}{c}\text { Random } \\
(\%)\end{array}$ & $\begin{array}{c}\text { Decoys } \\
(\%)\end{array}$ \\
\hline 1 & 11,16 & Model 1 & $\begin{array}{c}\text { Hyd, HBA, HBA, HBA, HBA, } \\
\text { HBD }\end{array}$ & 73 & 50 & 15 & 10 \\
\hline 2 & $2,3,13$ & Model 2 & Hyd, Ar, Ar, Ar, HBA, HBA & 10 & 0 & 5 & 0 \\
\hline 3 & 9,15 & Model 3 & Hyd, hyd, HBA, HBA & 66 & 75 & 8 & 39 \\
\hline 4 & 8,9 & Model 4 & $\begin{array}{c}\text { Hyd, Hyd, HBA, HBA, HBA, } \\
\text { HBD }\end{array}$ & 54 & 25 & 5 & 0 \\
\hline 5 & 24,27 & Model 5 & Hyd, Hyd, Hyd, HBA & 60 & 50 & 7 & 31 \\
\hline 6 & 4,13 & Model 6 & Hyd, Hyd, HBA, HBD & 20 & 0 & 13 & 30 \\
\hline 7 & $5,6,11$ & Model 7 & Hyd, Ar, HBA, HBA & 80 & 25 & 2 & 9 \\
\hline 8 & 4,5 & Model 8 & $\begin{array}{c}\text { Hyd, Hyd, Hyd Ar, HBA, HBA, } \\
\text { HBA, HBA, HBD }\end{array}$ & 7 & 0 & 3 & 0 \\
\hline 9 & 15,20 & Model 9 & Hyd, HBA, HBA, HBA & 83 & 50 & 15 & 36 \\
\hline 10 & 16,22 & Model 10 & $\begin{array}{c}\text { Hyd, Hyd, HBA, HBA, HBA, } \\
\text { HBA }\end{array}$ & 23 & 0 & 3 & 6 \\
\hline 11 & 6,23 & Model 11 & Hyd, Hyd, HBD, HBA, HBA & 43 & 50 & 0 & 8 \\
\hline 12 & 10,12 & Model 12 & Hyd, HBA, HBA, HBD & 30 & 0 & 8 & 0 \\
\hline
\end{tabular}




\subsection{Virtual Screening}

Virtual screening (VS) is an effective method, used for finding novel and potent active molecules (i.e. hits) as starting points for medicinal chemistry (Lavecchia \& Giovanni, 2013). In our study, sequential filtration was performed prior to virtual screening. In order to find out the druggability of $\sim 17$ million compounds (compounds from all four databases; Chembridge, Maybridge, NCI and ZINC) all compounds were filtered as per Lipinski's rules. This rule states that molecules which possess drug-like properties must contain molecular weight $<500, \log \mathrm{P}<5$, hydrogen bond acceptor $<5$ and hydrogen bond donor $<10$ otherwise these molecules have poor absorption or permeation (Lipinski, 2004). After Lipinski filtration, 12 million compounds were retrieved and subjected to active descriptors-based filtering. For this purpose, 2D descriptors of experimentally reported inhibitors such as MW (365-500 a.m.u), topological polar surface area (36-123), HBD (0-3), HBA (1-7), Slog P (2-7), formal charge (0-1) and number of rotatable bonds (2-11) were calculated by MOE2019.01 software (Table S1). The descriptor-based filtration provided 5 million compounds which were further subjected to pharmacophore-based virtual screening. The validated pharmacophore model was used for this screening and yielded 42,000 hits. Moreover, these hits were evaluated for statistical analysis via calculation of the Enrichment Factor (EF) and ROC (AUC) curve.

\subsection{Statistical Analysis (Enrichment factor and ROC CURVE)}

The receiver operating curve is defined as a graphical plot of rate of true positives (sensitivity) versus the rate of false-positive (1-specificity) (Empereur-mot et al., 2015) as shown in Figure 8, it is an effective method for the evaluation of virtual screening results. It is capable of retrieving 
both the information related to active and inactive and independent on the number of actives. The Area under curve (AUC) value of the best pharmacophore model is 0.67 indicating the reliability of the generated pharmacophore model.

Enrichment factor $(\mathrm{EF})$ is another statistical method commonly used for the assessment of virtual screening results (Bender \& Glen, 2005). The efficiency of docking and scoring function can also be estimated through the calculation of enrichment factors at a different percentage of databases. EF used to access the discriminatory power of the constructed pharmacophore model to rank known active ligands in the top of the list. In this study, according to EF calculation, most of the known actives lie in $50 \%$ of the database which contained approx. 21,000 hits that were further evaluated for the Tanimoto coefficient analysis.

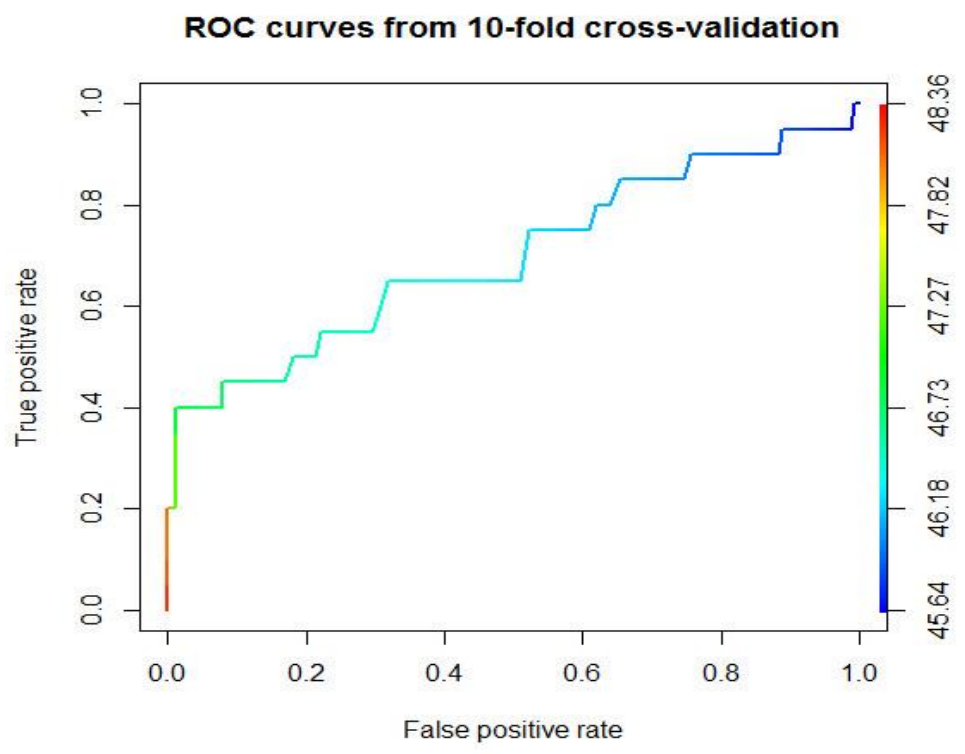

Figure 8. Receiver Operating Curve (ROC), a graphical plot between sensitivity (rate of true positive) versus 1Specificity (rate of a false negative). 


\subsection{Tanimoto Coefficient Analysis}

The 21,000 hits obtained after statistical analysis (EF and ROC Curve) were subjected to Similarity index calculation. The most potent inhibitors (Compound 5, 6, 11) which were previously used in the generation of pharmacophore models were used as a query molecule for Tanimoto similarity searching at different cuts off range 0.6-0.8. Initially, several conformations against the query compounds were generated then shape-based overlapping takes place with respect to the query compound. Subsequently, $0.6-0.8$ cut off was set to filter the obtained hits $(21,000)$ and finally, 4000 compounds were present in the predefined cut off the range. These 4000 compounds were further evaluated by molecular docking studies.

\subsection{Molecular Docking}

Molecular docking studies were performed to explore the binding modes of screened virtual hits. To evaluate the accuracy of docking software's redocking experiments were performed. The redocking results revealed that the top-ranked poses predicted by Auto Dock 4.2 were like the crystal binding pose of reference ligand. The root means square deviation (RMSD) of regenerated

crystal pose by AutoDock was $1.3 \AA$ indicating the reliability of our docking protocol. Re-docked results were summarized in Table 3 and Figure 9. Therefore, AutoDock 4.2 was used for predicting the binding mode of shortlisted virtual hits and PDB ID: 2AZ5 was used as a molecular model in docking studies. 
Table 3. Re-docking experiments of a complex structure of TNF- $\alpha$ with bound inhibitor SPD307 on different software.

\begin{tabular}{ccc}
\hline S.no & Docking Method & RMSD \\
\hline & Auto Dock 4.2 & $1.3 \AA$ \\
1 & GOLD & $11.9 \AA$ \\
2 & MOE-Dock & $9.6 \AA$ \\
3 & Open Eyes Fred & $4.9 \AA$ \\
4 & Surflex-Dock & $10.3 \AA$ \\
\hline
\end{tabular}

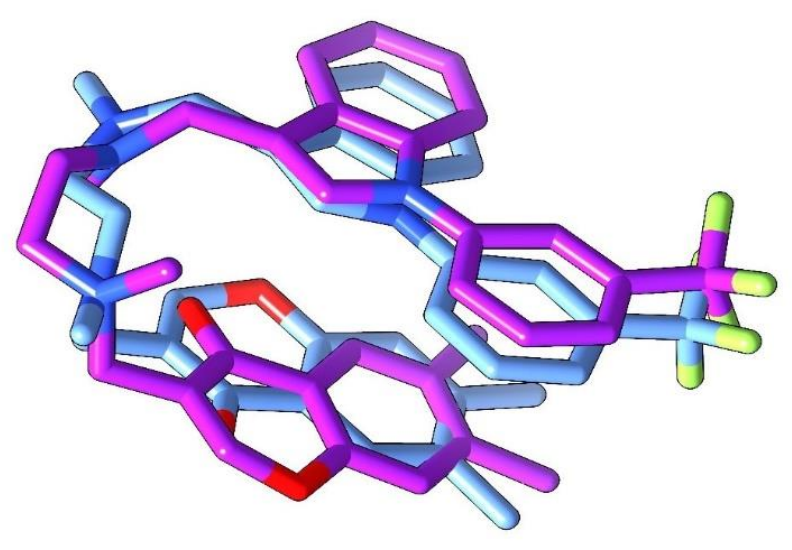

Figure 9. The redocked pose of 307 (bound inhibitor of TNF- $\alpha$ PDB I.D: 2AZ5). The magenta color shows the extracted pose of SPD307 while cyan is the best re-docking posed obtained by Auto Dock 4.2.

A total of 4,000 compounds were docked into the binding pocket of TNF- $\alpha$ protein. Top-ranked 480 compounds were selected based on their good docking score and further subjected to visual inspection. All the compounds were then analyzed visually for interactions and their binding strengths via MOE2019.01 using the crystal bound ligand's interactions as reference. After visual 
analysis 48 virtual hits showed good binding interaction with the hotspot residues of TNF- $\alpha$. All the selected compounds belonged to the ZINC database. The criteria for selecting the most promising leads were based on their good pharmacophore fit score, dock score and strong binding interaction with the hotspot residues of TNF- $\alpha$ protein. Out of 48 hits, only 6 compounds were fulfilling the above-mentioned criteria and considered as promising leads compounds (Table 4). The pharmacophore mapping, docking score and visual inspection of these predicted lead candidates showed that they might act as novel and potent inhibitors of TNF- $\alpha$.

Table 4. Dock and pharmacophore fit score of selected virtual leads.

$\begin{array}{llll}\text { Compound Name } & \begin{array}{c}\text { Docking Score } \\ \text { Pharmacophore }\end{array} & \text { Fit } & \\ \text { Score }\end{array}$


The visual analysis showed that the all the selected leads showing significant binding pattern with the crucial active site residues of TNF- $\alpha$ such as the top-ranked pose of compound 1 with high dock score $-8.45 \mathrm{kcal} / \mathrm{mol}$ (ZINC058418961) showed that it stabilizes itself by mediating two strong hydrogen bonds with oxygen atom of Gly121 A and another hydrogen bond with the amide nitrogen of Leu120A respectively. Compound 1 further stabilizes in the TNF binding pocket by showing some hydrophobic interaction with Leu57A, Leu57B, Tyr59A, Tyr59B and Gln61 of chain A. Quinoline ring of compound 1 showing pie-stacking interaction with the benzene ring of Tyr59 of chain B. Therefore, compound 1 accommodating itself with the simultaneous establishment of hydrogen bonds and hydrophobic interactions with hot spot residues proving its ability to inhibit TNF- $\alpha$.

The active site of TNF- $\alpha$ consists of many tyrosine residues and compound 2 (dock score -7.50 $\mathrm{kcal} / \mathrm{mol}$ ) (ZINC09402309) established itself by interacting with these tyrosine residues. The topranked pose of this compound showed several hydrophobic interactions with tyrosine residues of both chains A and B; Tyr59, Tyr119, and Tyr151 respectively. Additionally, compound 2 also involved in hydrophobic interaction with Leu57 and Gln61 of chain B. Moreover, this compound exhibit two hydrogen bonds with amide nitrogen and the oxygen atom of Gly121 of chain A. Furthermore, the hydroxyl group of the Tyr151A is involved in hydrophobic as well as hydrogen bonding interaction.

Compound 3 (ZINC04502991) has shown good dock score $(-7.45 \mathrm{kcal} / \mathrm{mol})$ and resided in the binding pocket of TNF protein by establishing several hydrogen bonds and few hydrophobic 
contacts. As the binding site of TNF- $\alpha$ is largely hydrophobic due to the presence of many tyrosine residues such as Tyr59, Tyr119 and Tyr 151. These tyrosine residues of both the chains are involved in hydrophobic interaction with the ligand molecule. Besides these interactions, this compound stabilizes itself inside the binding pocket by mediating four hydrogen-bonding interactions with Ser60A, Gln61B, Tyr119A, and Leu120A. The two hydrogen bonds were observed between the oxygen atom of the ligand molecule that is attached to Sulphur and with the backbone amide nitrogen of Ser60A and Leu120A. Thiadiazol ring of ligand molecule mediates two hydrogen bonds in bidentate fashion with the carbonyl group of Tyr119A and with the backbone amide nitrogen of Gln61 of chain B. The strong hydrogen bonding with crucial residues provides stability to the protein and makes this compound as a strong lead candidate to inhibit TNF- $\alpha$.

Another lead compound 4 (ZINC29558932) with docks core of $-7.10 \mathrm{kcal} / \mathrm{mol}$ mainly interacts with the protein's binding site by hydrophobic interaction. Benzene ring of ligand molecules showed intermolecular interaction with the methyl group of Tyr59A and the aliphatic chain of Leu57A. Moreover, this benzene ring also mediates $\square$ stacking interactions with the benzene ring of Tyr59B. Pyridine ring of compound 4 showed hydrophobic interaction with Tyr119 residue of chains $\mathrm{A}$ and $\mathrm{B}$ respectively. Furthermore, the pyrrolidine ring of compound exhibiting hydrophobic interaction with Tyr151 and Gln61 of chain A. Dioxin ring of ligand also interacts hydrophobically with the tyrosine (Tyr59 and Tyr151) residues of chain B.

Previously, it was reported that hydrophobic interaction plays an important role in accommodating ligands within the active site of TNF- $\alpha(\mathrm{He}, 2005)$. Compound 2 and compound 6 share a similar binding mode of hydrophobic interactions with the several tyrosine residues in the binding pocket 
of TNF- $\alpha$. Similarly, compound 5 (ZINC73690990) (dock score $-6.92 \mathrm{kcal} / \mathrm{mol}$ ) interacts with the TNF- $\alpha$ via hydrophobic as well as hydrogen bonding interaction. Fluorobenzene ring of ligand

interacts hydrophobically by Tyr59, Gln61, Tyr119, and Tyr151 of chain A. Leu57A, Tyr119B, and Tyr59 of chain B also involved in hydrophobic interactions with this compound. The oxygen atom of ligand molecule forming a hydrogen bond with the backbone amide nitrogen of Gly 121 of chain A. Another hydrogen bonding has been observed between the isoxazole ring of ligand molecule with the backbone amide nitrogen of Gly121 of chain B.

In the case of compound 6 (ZINC11915498) with dock score $-6.73 \mathrm{kcal} / \mathrm{mol}$, it stabilizes itself by mediating the number of hydrophobic interactions. The Chlorobenzene ring of the compound interacts hydrophobically with Tyr59B and Gln61B. Similarly, the Cyclohexane ring of ligand showed hydrophobic interaction with the aromatic ring of Tyr119A. Additionally, Tyr119 is making pie stacking interaction with the isoxazole ring of the ligand molecule. The oxygen atom in ligand molecule exhibiting a hydrogen bonding interaction with backbone amide nitrogen of Gly121 of chain A. From the docking analysis of lead compounds, it was revealed that all the compounds showed good binding pattern and interaction within the binding pocket by mediating several hydrogen bonding and hydrophobic interactions (Figure 10) showing their ability to inhibit TNF- $\alpha$ at nanomolar level. 

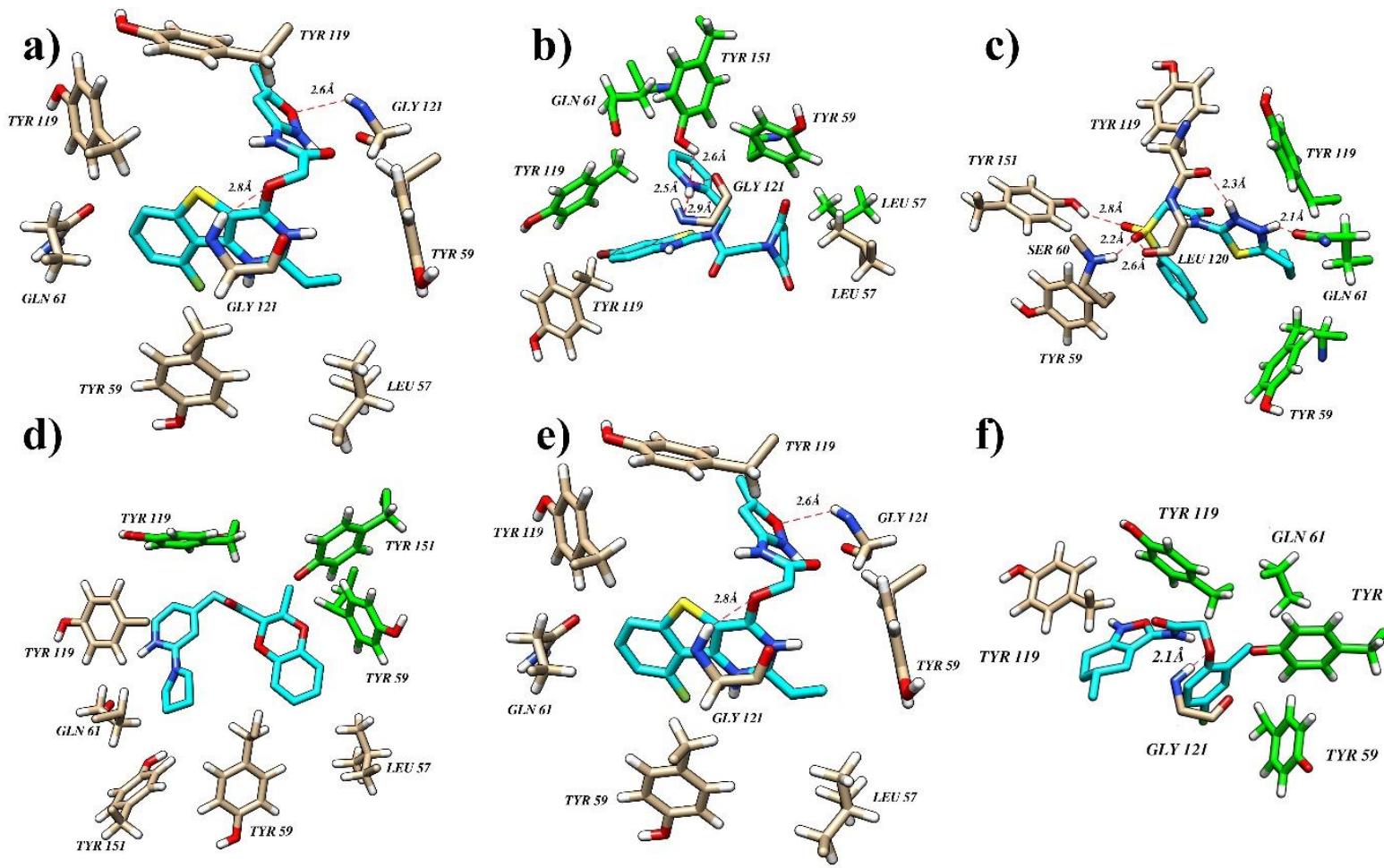

f)
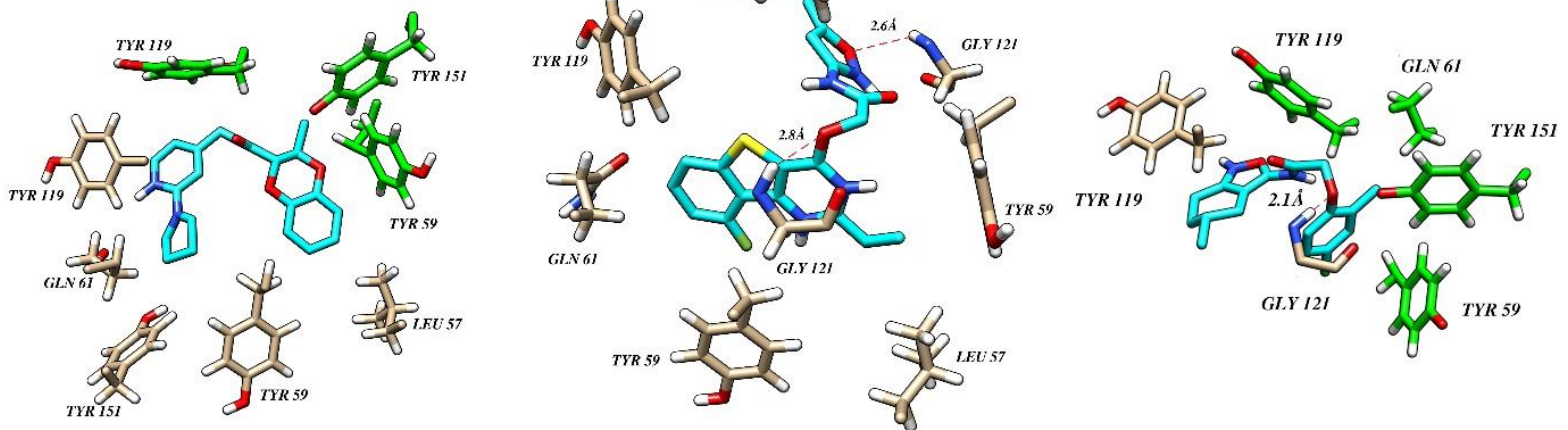

Figure 10. Dock poses of virtual hits: ZINC058418961, ZINC09402309, ZINC04502991, ZINC29558932, ZINC73690990, ZINC11915498 are shown in panel (a-f) in alphabetical order. TNF- $\alpha$ hotspot residues are represented as thick khaki sticks (chain A), thick green sticks (Chain B) and cyan color represented the ligand's structure. The dash red lines show the hydrogen bonding interaction.

\subsection{Molecular Dynamic Simulation}

Molecular dynamics simulation is an important tool for the validation of experimentally welldefined biological activities through finding the binding interactions between protein and its inhibitor (Durrant \& McCammon, 2011). The method's distinctive feature in a drug design context 
is its ability to analyze protein-ligand systems in aqueous solution at physiological conditions, thus maximizing resemblance of the computational model to real physical-chemical conditions of the

environment realized in an experiment. Also, the dynamics of the system at molecular scales can be investigated, the property that is impossible to obtain in all other computational approaches in drug design. It is known that water and ions play a crucial role in the structure and, especially, the dynamics of proteins. It is, therefore, possible that the results obtained in virtual screening and docking could be corrected by the presence of water and ions at room temperature.

We have examined the structural or conformational changes in the active site of TNF- $\alpha$ protein against its respective inhibitors. MD Simulation was performed for the five complexes including apo-protein, reference compound, and three lead molecules. It represents the overall stability of each complex during the 100 nanoseconds simulation run. The stability of all the complexes was studied by Root mean square deviation (RMSD), Root-mean-square fluctuation (RMSF), Radius of gyration $(\mathrm{Rg})$ and the number of hydrogen bonds.

\subsection{Root mean square deviation (RMSD)}

To rationalize the reliability of our sampling protocol and to determine the stability of protein structure during the $100 \mathrm{~ns}$ simulation, the RMSD curves of the backbone atoms of protein were calculated and compared with its initial coordinates. All the five complexes showed variable deviations during simulation in the backbone of TNF- $\alpha$. The RMSD of all complexes was calculated by using the g_rms tool of Gromacs software. After the equilibration phase, the Simulation system of reference compound rose continuously from $0.34-0.42 \mathrm{~nm}$ and finally, after 40ns the system was stable at $0.39 \mathrm{~nm}$ throughout the simulation. RMSD value of virtual hit 
compound 1 rose continuously in the first 35ns, increasing from $0.37-0.39 \mathrm{~nm}$ and after $40 \mathrm{~ns}$ attained stability with an average of $0.37 \mathrm{~nm}$ during the entire simulation. In all simulation systems,

compound 2 and 3 reach equilibrium early and show less fluctuation during 100ns simulation. A small fluctuation was observed in compound 3 between 12-33ns that was slightly increased in rmsd $0.37 \mathrm{~nm}$, then slowly decreased and stabilized at $0.36 \mathrm{~nm}$. In the light of above analysis, the simulated confirmation of all the system has small deviation from their initial conformation, and RMSD values of apo, reference and compound 1,2,3 were in a stable range after MD simulation such as $0.44 \mathrm{~nm}, 0.4 \mathrm{~nm}, 0.37 \mathrm{~nm}, 0.34 \mathrm{~nm}$, and $0.36 \mathrm{~nm}$ respectively (Figure 11).

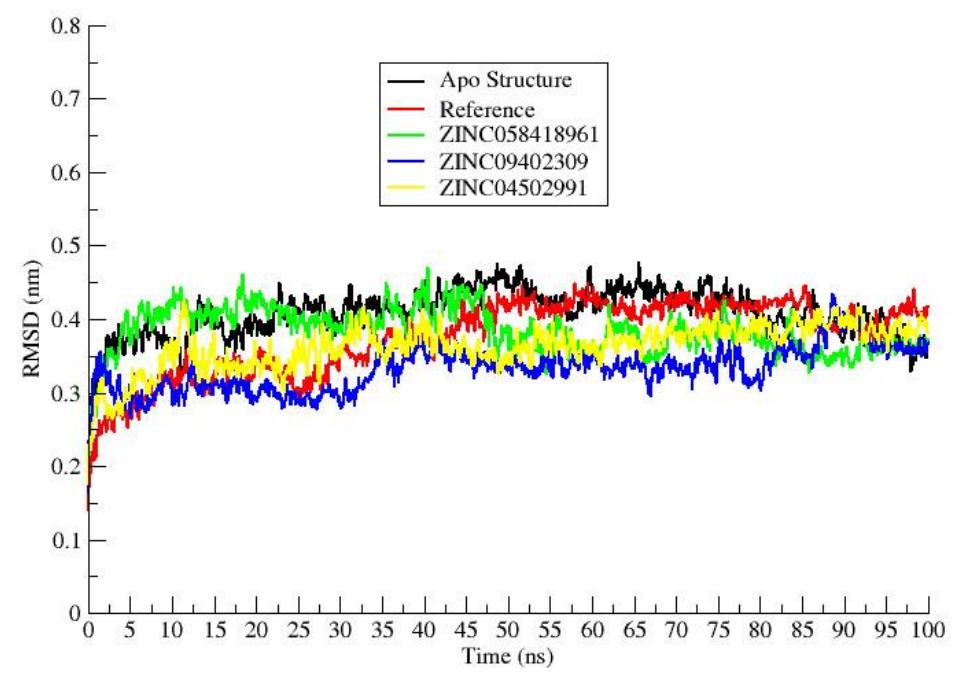

Figure 11. a) The Root Mean Square Deviation (RMSD) plots all complexes over the course of 100ns simulation.

\subsection{Root Mean Square Fluctuation (RMSF)}

In order to determine the ligand-binding effect on the stability of protein structure RMSF analysis was carried out of every system over the 100ns MD simulation depicted in Figure 12. The RMSF 
of backbone atoms was calculated by the g_rmsf tool implemented in Gromacs software. The RMSF of backbone atoms (N, C $\alpha$, and C) of TNF- $\alpha$ in each system were examined and calculated by MD trajectories. The active site residues (Leu57 A\& B, Tyr59 A \& B, Ser60 A \& B, Glu61 A \& B, Tyr119 A \& B, Leu120 A \& B, Gly121-122 A \& B, Tyr151 A \& B) in all ligand-protein complexes were showing stability by maintaining the average RMSF value of $0.1 \pm 0.15 \mathrm{~nm}$ indicating that residues seem to be more rigid in the presence of ligands. The average fluctuation of all complexes was found to be $0.015 \mathrm{~nm}$ (reference), $0.016 \mathrm{~nm}$ (compound 1), $0.023 \mathrm{~nm}$ (compound 2), and 0.016nm (compound 3) respectively. Most of the higher fluctuations were observed in loop regions of chain A (residue no. 21-26, 32-40,104-112) and chain B (residue no.168-174, 232-236) because these loops are a most flexible part of the protein.

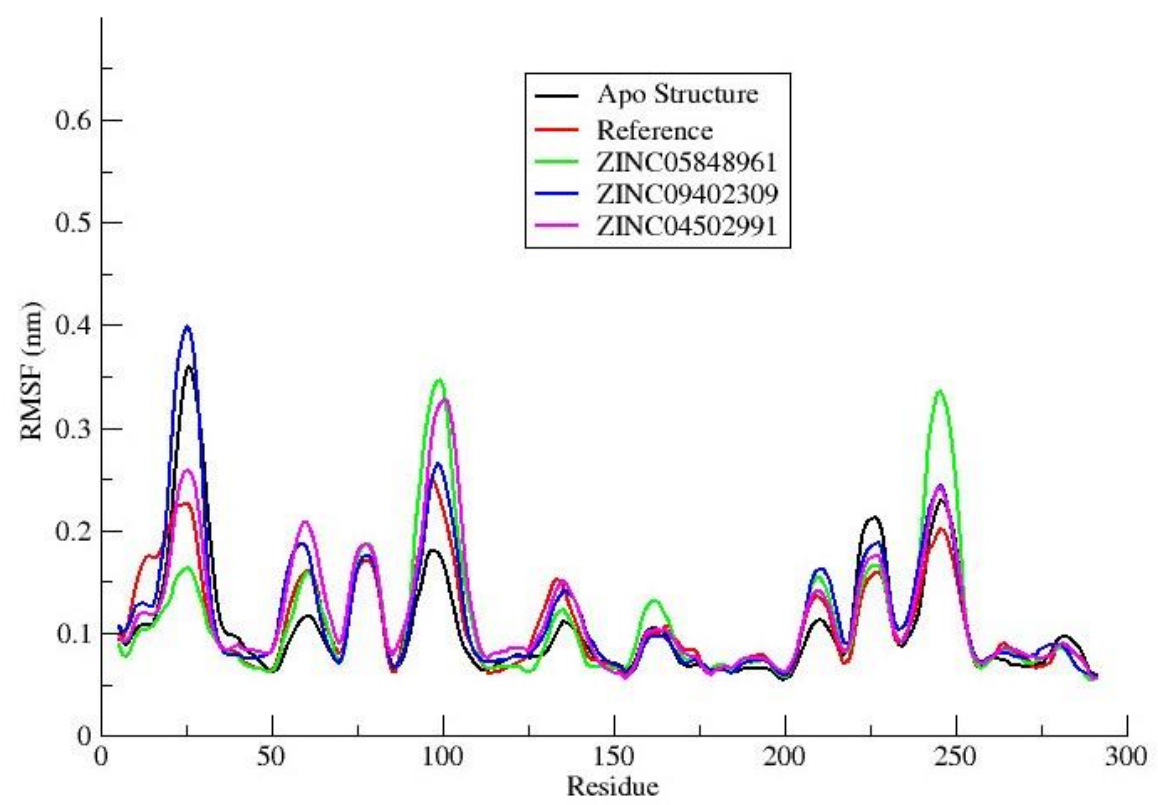

Figure 12. Comparative backbone RMSF of all the complexes. The visual depicts a significant difference in the RMSF profile of each complex. 


\subsection{Radius of gyration $(\mathrm{Rg})$}

The radius of gyration measures the compactness level of protein structure and lower Rg values show that the structure is more compact and stable showing lower conformational entropy (Lobanov, Bogatyreva \& Galzitskaya, 2008). We performed the Rg analysis of all complexes and apoprotein by using the g_rms tool implemented in Gromacs software. Figure 13 demonstrates the major fluctuation of apoprotein starting from 2.07A and slightly fluctuating 2.04 to 2.03 after $6.5 \mathrm{~ns}$ attained stability at $2.03 \AA$ for the entire $100 \mathrm{~ns}$ simulation. In contrast, the Rg values of the reference compound, compound 1,2, 3 were showed structural stability at 2.02A, 2.01 A, 2.02 A and 2.0 A respectively. The values of $\mathrm{Rg}$ for reference, compound 2 and 3 tended to be stable and fluctuated constantly till $5 \mathrm{~ns}$ and converged throughout the simulation, but compound 1 showed high fluctuations till $8 \mathrm{~ns}$ and then became stable during the entire simulation. In comparison to apoprotein, the TNF complex with its inhibitors showed low Rg values indicating less entropy/disorders in the TNF receptor when bound with the ligand complexes. 


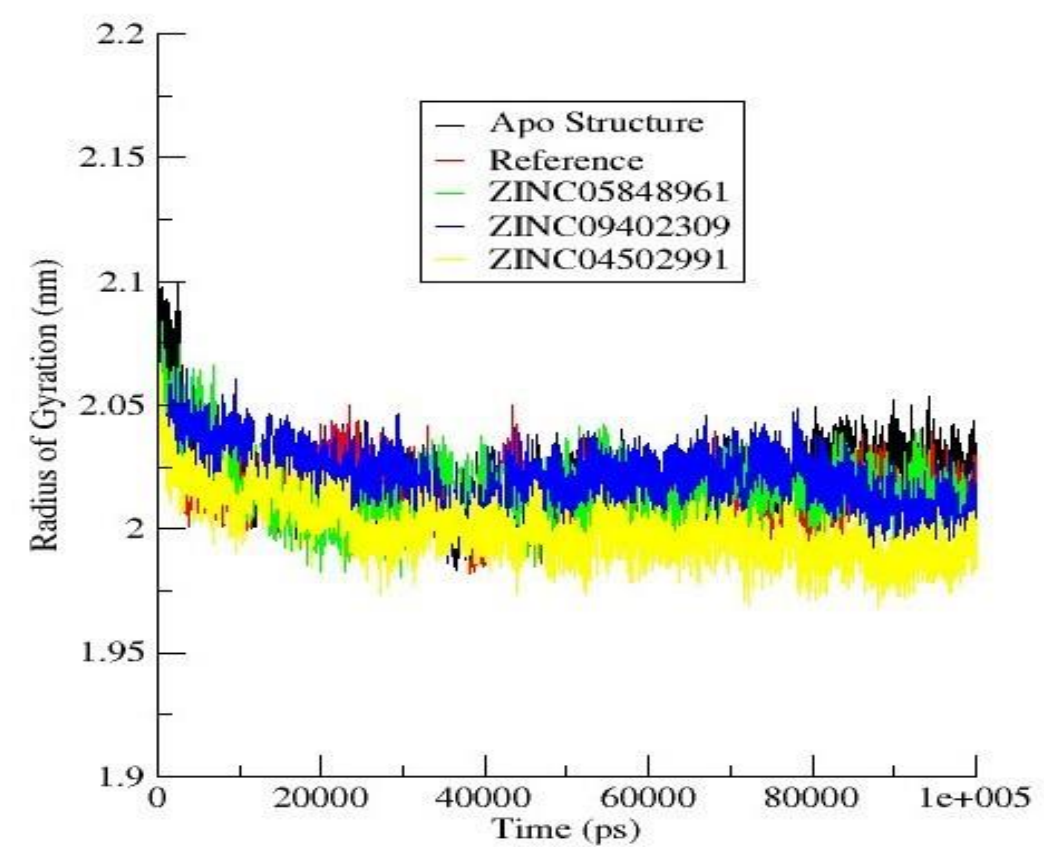

Figure 13. showing $\operatorname{Rg}$ of every complex throughout the $100 \mathrm{~ns}$ simulation.

\subsection{Hydrogen bond Analysis}

Hydrogen bonds play a significant role in protein stability and it helps in increasing the stability of protein-ligand complexes (Fu, Zhao \& Chen, 2018). The number of hydrogen bonds was determined by using the g_hbond tool in Gromacs. The predicted binding pattern of all lead compounds was well correlated in docking as well as in MD analysis. As TNF- $\alpha$ has a largely hydrophobic pocket, in case of reference compound no hydrogen bonding was observed and it resides within the TNF binding pocket by interacting hydrophobically with the hot spot residues. Moreover, we identified three lead compounds ZINC05848961 (Compound 1), ZINC09402309 (Compound 2), ZINC04502991 (Compound 3), that were showing several hydrophobic as well as good hydrogen bonding interaction within the TNF- $\alpha$ binding pocket. The compound 1 forming two strong hydrogen-bonding interactions with the oxygen atom of Gly121A and one with the backbone amide nitrogen of Leu120A. However, the first hydrogen bond formed between 
Gly121A was stable after 12ns while the second hydrogen bond with this residue was not stable and fluctuated continuously throughout the simulation, and the third hydrogen bond with Leu120A was lost during the entire simulation.

In the case of Compound 2, it forms two hydrogen bonds with amide nitrogen of Gly121 of chain A and a third one with the hydroxyl moiety of the Tyr151A. Among these three hydrogen bonds, only one with Gly121A is stable after 5ns and fluctuated substantially throughout the simulation, while the second hydrogen bond (Gly121A) was lost after 40ns simulation and again re-appeared at $90 \mathrm{~ns}$ of simulation. The third hydrogen bond between Tyr151A and compound 2 was completely lost during the 100ns simulation. The compound 3 stabilizes itself by forming five hydrogen bonds with Ser60A, Gln61B, Tyr119A, and Leu120A and Tyr151A, but only Ser60A and Leu120 were stable during the later stages of molecular dynamics simulation. The hydrogen bonds between Gln61B, Tyr119A, Tyr151A were not stable and fluctuated continuously during the entire simulation. The hydrogen bonding interaction of all compounds was depicted in Figure 14. Moreover, all the compounds showed good hydrophobic interactions with crucial tyrosine residues of chain A \& B (Tyr59, Tyr119, and Tyr151) and Leu57 of chain A and were well correlated with docking results. The detailed structural understandings and knowledge of these predicted virtual hits proved that it can be effective in autoimmune therapies. Moreover, in vivo or in vitro experimental assays are required to check the inhibitory activities of these lead compounds. 


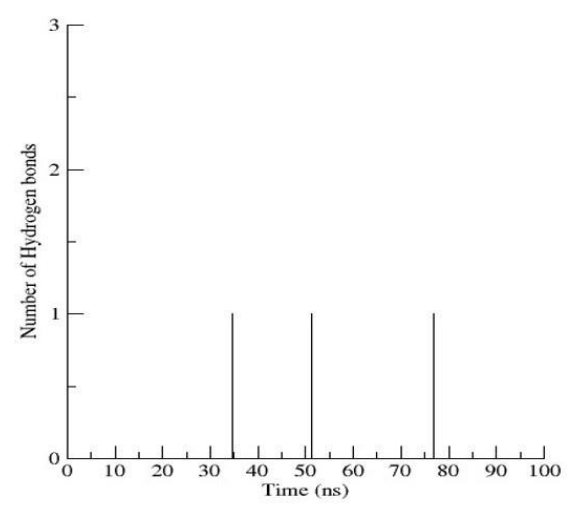

Reference Compound

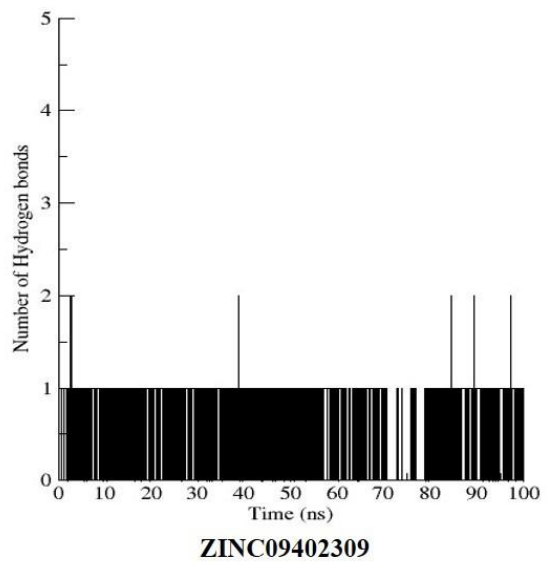

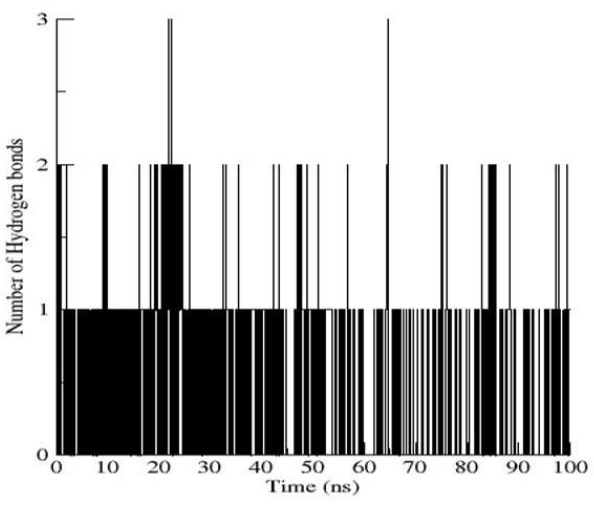

ZINC05848961

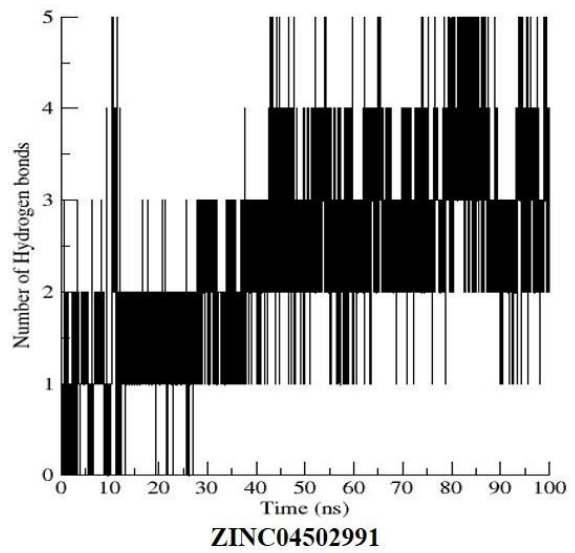

Figure 14. Stability interpretation of all complexes using intermolecular hydrogen bonding pattern as a function of time.

\subsection{Pharmacological Prediction of Selected Hits}

All the selected hits were evaluated for their physicochemical and pharmacokinetic properties using the Swiss ADME online server and results were shown in Figure 15 and Table $5 \&$ 6. The prediction of drug-likeness was also calculated based on the Lipinski, Ghose, Veber, Egan, Mugguae rules and bioavailability scores. According to Lipinski rules the good absorption or permeation of a molecule is more likely when MW $<5 \mathrm{~g} / \mathrm{mol}, \operatorname{LogP}<5, \mathrm{HBD}<5$ and $<10$ HBA atoms. Veber rules state that a molecule is considered to be drug-like if they contain $<10$ rotatable 
bonds and TPSA $\leq 140 \mathrm{~A}^{2}$. The Ghose filter characterizes the molecules based on their physicochemical properties such as $\log \mathrm{P}$ (between -0.4 and 5.6), Molar refractivity (between 30 and 130), and a total number of atoms (between 20 and 70). Egan filter provides a prediction of drug absorption properties based on the membrane permeability. The Muegee model refers to some functional groups as pharmacophoric points (amide, amine, alcohol, sulfone, ketone sulfonamide, carbamate, carboxylic acid, guanidine, amidine, ester, and urea) to classify drug-like and nondrug-like molecules. These pharmacophoric points provide significant interactions with the target receptor. Considering above mentioned filters, all the predicted hits are compatible with all druglikeness parameters and have good oral bioavailability profiles (0.55) according to Egan et al. (Egan, Merz, \& Baldwin, 2000) as shown in Table 6 \& 7. It indicated that all the compounds were suitable and well absorbed in the human body.

Moreover, $\log \mathrm{P}$ and $\log \mathrm{S}$ values were also determined by the Swiss ADME online server. According to the calculated $\log \mathrm{P}$ values, it can be concluded that all the compounds have good lipid solubility which will facilitate the drug interaction with the biological membranes and to be used for bioactivity generation. The ZINC04502991 was considered as least lipophilic due to its low $\log$ P-value. (iLOGP $=$-1.66). The compounds water solubility was predicted via the ESOL method and $\log \mathrm{S}$ values were in the range of -5.31-3.71 indicating that all compounds were moderately water-soluble. Furthermore, the synthetic accessibility score (SA) refers to the estimation of ease of synthesis of compounds and the SA score of all the compounds (3.77-2.48) was in the range of easy synthetic accessibility. PAINS (Pan-assay interference compounds) are those chemical compounds that give false-positive results in high-throughput screening and capable of interacting with various biological targets instead of interacting with one specific target 
(Baell, Ferrins, Falk \& Nikolakopoulos, 2013). In Swiss ADME evaluation, no PAINS alert was observed representing the specific nature of compounds.

Table 5. Predicted Physiochemical properties of the selected virtual hits compounds by Swiss ADME.

\begin{tabular}{llllllllllll}
\hline \multicolumn{10}{c}{ Physiochemical Properties of Hits } \\
\hline \multirow{2}{*}{ Ligands } & $\begin{array}{l}\text { Molecular } \\
\text { Formula }\end{array}$ & $\begin{array}{l}\text { Molec } \\
\text { ular } \\
\text { Weigh } \\
\text { t }\end{array}$ & $\begin{array}{l}\text { Hea } \\
\text { vy }\end{array}$ & $\begin{array}{l}\text { Ato } \\
\text { ms }\end{array}$ & $\begin{array}{l}\text { ic } \\
\text { Heavy } \\
\text { Atoms }\end{array}$ & $\begin{array}{l}\text { Frac } \\
\text { tion } \\
\text { Csp }\end{array}$ & $\begin{array}{l}\text { Rotata } \\
\text { ble } \\
\text { bonds }\end{array}$ & $\begin{array}{l}\text { Hydrog } \\
\text { en bond } \\
\text { Accepto } \\
\text { r }\end{array}$ & $\begin{array}{l}\text { Hydro } \\
\text { gen } \\
\text { bond } \\
\text { Donor }\end{array}$ & $\begin{array}{l}\text { Molecular } \\
\text { refractivit } \\
\text { y }\end{array}$ & TPSA \\
\hline & & & & & & & & & & \\
ZINC05848961 & C19H15BrN2O3 & 399.24 & 25 & 16 & 0.11 & 6 & 4 & 1 & 99.52 & 68.29 \\
ZINC09402309 & C19H15BrN4O3S & 459.32 & 28 & 15 & 0.21 & 6 & 5 & 0 & 113.14 & 111.71 \\
ZINC04502991 & C14H17N3O3S2 & 339.43 & 22 & 11 & 0.36 & 7 & 5 & 1 & 86.38 & 125.64 \\
ZINC11915498 & C17H19CIN2O3 & 334.8 & 23 & 11 & 0.41 & 5 & 4 & 1 & 89.04 & 64.36 \\
ZINC29558932 & C20H23N3O3 & 353.41 & 26 & 12 & 0.4 & 5 & 4 & 1 & 101.66 & 63.69 \\
ZINC73690990 & C18H15FN4O3S & 386.4 & 27 & 18 & 0.22 & 6 & 7 & 1 & 100 & 118.38 \\
\hline
\end{tabular}

Table 6. Predicted Drug-likeness properties of the selected hits compounds by Swiss ADME.

\begin{tabular}{lllllllllll}
\hline Ligands & $\begin{array}{l}\text { Lipinski } \\
\text { Violatio }\end{array}$ & $\begin{array}{l}\text { Ghose } \\
\text { Violati } \\
\text { on }\end{array}$ & $\begin{array}{l}\text { Veber } \\
\text { Violatio } \\
\mathbf{n}\end{array}$ & $\begin{array}{l}\text { Egan } \\
\text { Violatio } \\
\mathbf{n}\end{array}$ & $\begin{array}{l}\text { Muegge } \\
\text { Violatio } \\
\mathbf{n}\end{array}$ & $\begin{array}{l}\text { Bioavail } \\
\text { ability } \\
\text { Score }\end{array}$ & $\begin{array}{l}\text { Synthetic } \\
\text { accessibil } \\
\text { ity }\end{array}$ & $\begin{array}{l}\text { PAI } \\
\text { NS } \\
\text { Alert }\end{array}$ & $\begin{array}{l}\text { Log } \\
\text { P }\end{array}$ & $\begin{array}{l}\text { Log } \\
\text { S }\end{array}$ \\
\hline ZINC05848961 & 0 & 0 & 0 & 0 & 0 & 0.55 & 2.48 & 0 & 3.07 & -5.13 \\
ZINC09402309 & 0 & 0 & 0 & 0 & 0 & 0.55 & 2.94 & 0 & 2.56 & -4.06 \\
ZINC04502991 & 0 & 0 & 0 & 0 & 0 & 0.55 & 3.29 & 0 & 1.66 & -3.71 \\
ZINC29558932 & 0 & 0 & 0 & 0 & 0 & 0.55 & 3.7 & 0 & 2.63 & -3.82 \\
ZINC11915498 & 0 & 0 & 0 & 0 & 0 & 0.55 & 3.77 & 0 & 2.54 & -4.49 \\
ZINC73690990 & 0 & 0 & 0 & 0 & 0 & 0.55 & 3.5 & 0 & 3.03 & -4.83 \\
\hline
\end{tabular}

Human gastrointestinal absorption (GI), blood-brain barrier permeability and permeation of skin are important pharmacokinetic properties that indicate the ability of the human body to absorb the 
molecules (Berben et al., 2018). The boiled egg analysis of SwissADME was used to predict the bioavailability properties of all compounds.

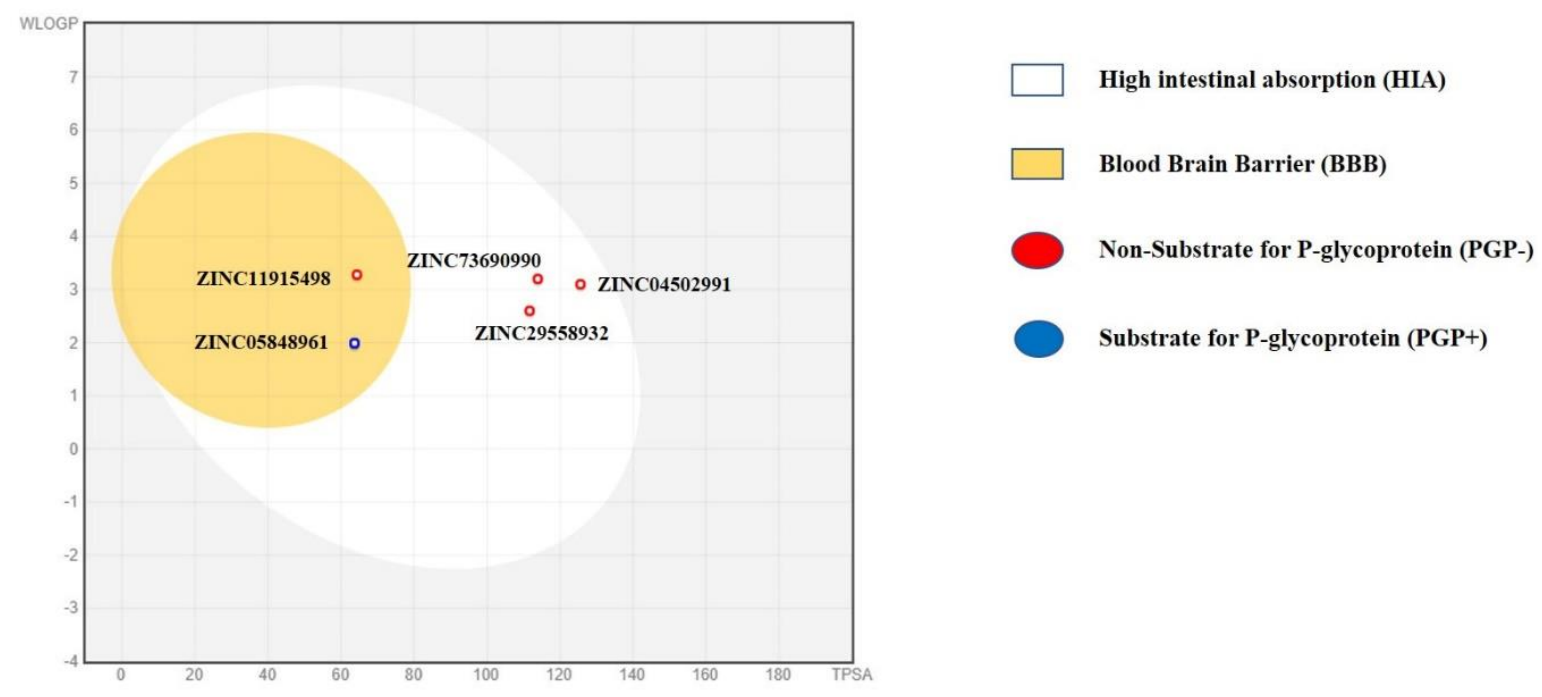

Figure 15. Boiled Egg Analysis (water partition coefficient (WlogP) vs. Topological polar surface area (TPSA) of selected lead compounds by SwissADME server.

The results of boiled egg analysis revealed that all the predicted compounds showed high GI absorption indicated that they are suitable for oral administration and ZINC05848961, ZINC11915498, ZINC29558932 were able to cross the blood-brain barrier due to their high lipophilic nature. ZINC09402309 (-7.45 cm/s), ZINC29558932 (-6.45 cm/s) and ZINC04502991($6.24 \mathrm{~cm} / \mathrm{s}$ ) were predicted to have less skin permeability while ZINC11915498 have high skin permeability $(-5.47 \mathrm{~cm} / \mathrm{s})$. P-glycoproteins (p-gp) are ATP driven efflux pumps involved in the transport of drugs in various organs and often responsible for drug resistance for anti-cancer drugs (Lin \& Yamazaki, 2003). All the predicted hits were non-substrate to P-gp except ZINC29558932. 
This proposed that the predicted have a lower chance of efflux out of the cell that will help in producing the maximal effect of the drug.

The cytochrome P450 enzymes family plays a vital role in the biotransformation of drugs. These enzymes are important determinants of drug metabolism which can lead to decrease pharmacological effects and drug toxicities (Jana \& Paliwal, 2007). The compound ZINC05848961, ZINC11915498, ZINC73690990 were acting as an inhibitor of all the isoforms of the CYP family (CYP1A2, CYP2C19, CYP2C9, CYP2D6, CYP3A4). ZINC09402309, ZINC04502991 is the inhibitor of all isoforms except CYP2D6, CYP3A4, CYP1A2, and CYP2C9). Most of the drugs are activated or inactivated by these enzymes, therefore, some biological assays will be needed to explore the activation and deactivation mechanism of these compounds by CYP isoforms (Table 7).

Table 7. Predicted Pharmacokinetic properties of the selected virtual hits compound by Swiss ADME.

\begin{tabular}{llllllllll}
\hline Ligands & $\begin{array}{l}\text { GI } \\
\text { absorptio } \\
\mathbf{n}\end{array}$ & $\begin{array}{l}\text { BBB } \\
\text { perm } \\
\text { eabilit } \\
\mathbf{y}\end{array}$ & $\begin{array}{l}\text { P-gp } \\
\text { Subst } \\
\text { rate }\end{array}$ & $\begin{array}{l}\text { CYP1A2 } \\
\text { inhibitor }\end{array}$ & $\begin{array}{l}\text { CYP2C19 } \\
\text { inhibitor }\end{array}$ & $\begin{array}{l}\text { CYP2C9 } \\
\text { Inhibitor }\end{array}$ & $\begin{array}{l}\text { CYP2D6 } \\
\text { Inhibitor }\end{array}$ & $\begin{array}{l}\text { CYP3A4 } \\
\text { Inhibitor }\end{array}$ & $\begin{array}{l}\text { Log Kp } \\
\text { Skin } \\
\text { permeati } \\
\text { on (cm/s) }\end{array}$ \\
\hline & & & & & & & & & \\
ZINC05848961 & High & Yes & No & Yes & Yes & Yes & Yes & Yes & -5.65 \\
ZINC09402309 & High & No & No & Yes & Yes & Yes & No & Yes & -7.56 \\
ZINC04502991 & High & No & No & Yes & Yes & Yes & No & No & -6.28 \\
ZINC05848961 & High & Yes & No & No & Yes & No & Yes & Yes & -6.45 \\
ZINC09402309 & High & Yes & Yes & Yes & Yes & Yes & Yes & Yes & -5.47 \\
ZINC04502991 & High & No & No & Yes & Yes & Yes & Yes & Yes & -5.84 \\
\hline
\end{tabular}

The selected hits were also analyzed for the toxicity profiles using Osiris DatawarriorV.5.0.0 software (Sander, Freyss, von Korff \& Rufener, 2015). According to the toxicity prediction (Table 8), there is no risk of being mutagenic, tumorigenic, reproductive and irritant for all compounds 
except ZINC11915498, which is highly mutagenic, tumorigenic, reproductive effect and low irritant. This compound can cross the BBB but did not violate the drug-likeness rules, also have high GIT absorption and skin permeation therefore due to high toxic properties it cannot be considered as TNF-inhibitor.

Table 8. Predicted Toxicity properties of the selected hits compounds by Osiris DataWorriers.

Toxicity Profiles of Selected Hits

\begin{tabular}{lllll}
\hline Ligands & Mutagenic & Tumorigenic & Reproductive effect & Irritant \\
\hline & & & & \\
ZINC05848961 & None & None & None & None \\
ZINC09402309 & None & None & None & None \\
ZINC04502991 & None & None & None & None \\
ZINC29558932 & None & None & None & None \\
ZINC11915498 & High & High & High & Low \\
ZINC73690990 & None & None & None & None \\
\hline
\end{tabular}

\section{Conclusion}

TNF- $\alpha$ is a multifunctional proinflammatory cytokine responsible for various pathological conditions and acts as a potential drug target to combat autoimmune and inflammatory disorders. In this study, we employed exhaustive computational drug design protocol including ligand-based pharmacophore mapping, virtual screening, docking, and MD simulation to develop potent smallmolecule TNF inhibitors. In this connection, the validated pharmacophore model was used as a three-dimensional query in the screening of four small molecules libraries (Chembridge, Maybridge, NCI, and ZINC). The subsequent validation and statistical analysis suggested that the generated pharmacophore model has a good ability to differentiate between active and decoys 
datasets. The pharmacophore screen hits were subjected to molecular docking studies to find out their binding modes with the target receptor. Molecular docking results suggested that the selected inhibitors have the potential to inactivate the TNF- $\alpha$ dimer by interacting with crucial residues by the formation of hydrogen bonds and hydrophobic interactions in a simultaneous fashion. Finally, six hits with diverse scaffolds were considered as lead candidates based on their highest binding affinity and good binding interaction with the target receptor. Moreover, in silico ADMET analysis suggested that all five leads possess good ADMET properties except ZINC11915498 due to its high predicted toxicity. Furthermore, MD simulation studies revealed that all three leads (ZINC05848961, ZINC09402309, ZINC04502991) showed good stability and remain in the binding pocket throughout the simulation. In conclusion, these leads have diverse scaffolds and can possibly act as starting points in further designing of potent small molecular inhibitors, but further experimental validation is required to prove their inhibitory activities.

\section{Acknowledgements}

The authors are grateful to the Higher Education Commission (HEC) for their Financial Support.

\section{Disclosure statement}

No potential conflict of interest was reported by the authors. 


\section{References}

- Abraham, M., Murtola, T., Schulz, R., Páll, S., Smith, J., Hess, B., \& Lindahl, E. (2015). GROMACS: High-performance molecular simulations through multi-level parallelism from laptops to supercomputers. SoftwareX, 1-2, 19-25.

- Adegbola, S., Sahnan, K., Warusavitarne, J., Hart, A., \& Tozer, P. (2018). Anti-TNF Therapy in Crohn's Disease. International Journal of Molecular Sciences, 19(8), 2244.

- Ajmal Shah, M., Khalil, R., Ul-Haq, Z., \& Panichayupakaranant, P. (2017). a-Glucosidase inhibitory effect of rhinacanthins-rich extract from Rhinacanthus nasutus leaf and synergistic effect in combination with acarbose. Journal of Functional Foods, 36, 325-331.

- Alexiou, P., Papakyriakou, A., Ntougkos, E., Papaneophytou, C., Liepouri, F., \& Mettou, A. et al. (2014). Rationally Designed Less Toxic SPD-304 Analogs and Preliminary Evaluation of Their TNF Inhibitory Effects. Archiv Der Pharmazie, 347(11), 798-805.

- Baell, J., Ferrins, L., Falk, H., \& Nikolakopoulos, G. (2013). PAINS: Relevance to Tool Compound Discovery and Fragment-Based Screening. Australian Journal of Chemistry, 66(12), 1483.

- Berendsen, H., Postma, J., van Gunsteren, W., DiNola, A., \& Haak, J. (1984). Molecular dynamics with coupling to an external bath. The Journal of Chemical Physics, 81(8), 36843690 .

- Berman, H. (2000). The Protein Data Bank. Nucleic Acids Research, 28(1), 235-242.

- Berben, P., Bauer-Brandl, A., Brandl, M., Faller, B., Flaten, G., \& Jacobsen, A. et al. (2018). Drug permeability profiling using cell-free permeation tools: Overview and applications. European Journal of Pharmaceutical Sciences, 119, 219-233.

- Bender, A., \& Glen, R. (2005). A Discussion of Measures of Enrichment in Virtual Screening: Comparing the Information Content of Descriptors with Increasing Levels of Sophistication. Journal of Chemical Information And Modeling, 45(5), 1369-1375. 
- Berg, T. (2003). Modulation of Protein-Protein Interactions with Small Organic Molecules. Angewandte Chemie International Edition, 42(22), 2462-2481.

- Bongartz, T., Matteson, E., \& Orenstein, R. (2005). Tumor necrosis factor antagonists and infections: The small print on the price tag. Arthritis \& Rheumatism, 53(5), 631-635.

- Bowie, J., Luthy, R., \& Eisenberg, D. (1991). A method to identify protein sequences that fold into a known three-dimensional structure. Science, 253(5016), 164-170.

- Buchwald, P. (2010). Small-molecule protein-protein interaction inhibitors: Therapeutic potential in light of molecular size, chemical space, and ligand binding efficiency considerations. IUBMB Life, 62(10), 724-731.

- Chan, F., Siegel, R., \& Lenardo, M. (2000). Signaling by the TNF Receptor Superfamily and T Cell Homeostasis. Immunity, 13(4), 419-422.

- Chan, D., Lee, H., Yang, F., Che, C., Wong, C., \& Abagyan, R. et al. (2010). StructureBased Discovery of Natural-Product-like TNF- $\alpha$ Inhibitors. Angewandte Chemie, 122(16), 2922-2926.

- Cheng, J., \& et al., e. (2004). Discovery and Structure-Activity Relationship of Coumarin Derivatives as TNF- $\alpha$ Inhibitors. Cheminform, 35(36).

- Choi, H., Lee, Y., Park, H., \& Oh, D. (2010). Discovery of the inhibitors of tumor necrosis factor-alpha with structure-based virtual screening. Bioorganic \& Medicinal Chemistry Letters, 20(21), 6195-6198.

- Clark, M., Cramer, R., \& Van Opdenbosch, N. (1989). Validation of the general purpose tripos 5.2 force field. Journal of Computational Chemistry, 10(8), 982-1012.

- Colovos, C., \& Yeates, T. (1993). Verification of protein structures: Patterns of nonbonded atomic interactions. Protein Science, 2(9), 1511-1519.

- Cortes, C., \& Vapnik, V. (1995). Support-vector networks. Machine Learning, 20(3), 273297.

- Daina, A., Michielin, O., \& Zoete, V. (2017). SwissADME: a free web tool to evaluate pharmacokinetics, drug-likeness and medicinal chemistry friendliness of small molecules. Scientific Reports, 7(1). 
- Davis, J. M., \& Colangelo, J. (2013). Small-molecule inhibitors of the interaction between TNF and TNFR. Future Medicinal Chemistry, 5(1), 69-79.

- Darden, T., York, D., \& Pedersen, L. (1993). Particle mesh Ewald: An N·log(N) method for Ewald sums in large systems. The Journal of Chemical Physics, 98(12), 10089-10092.

- Dhuru, S., Bhedi, D., Gophane, D., Hirbhagat, K., Nadar, V., \& More, D. et al. (2011). Novel diarylheptanoids as inhibitors of TNF- $\alpha$ production. Bioorganic \& Medicinal Chemistry Letters, 21(12), 3784-3787.

- Durrant, J., \& McCammon, J. (2011). Molecular dynamics simulations and drug discovery. BMC Biology, 9(1).

- Egan, W., Merz, K., \& Baldwin, J. (2000). Prediction of Drug Absorption Using Multivariate Statistics. Journal Of Medicinal Chemistry, 43(21), 3867-3877.

- Empereur-mot, C., Guillemain, H., Latouche, A., Zagury, J., Viallon, V., \& Montes, M. (2015). Predictiveness curves in virtual screening. Journal Of Cheminformatics, 7(1).

- Essmann, U., Perera, L., Berkowitz, M., Darden, T., Lee, H., \& Pedersen, L. (1995). A smooth particle mesh Ewald method. The Journal of Chemical Physics, 103(19), 85778593.

- EUGENE, V., SERGEY, A. \& GALA, V. 2010. ChemBridge. US private company.

- Fu, Y., Zhao, J., \& Chen, Z. (2018). Insights into the Molecular Mechanisms of ProteinLigand Interactions by Molecular Docking and Molecular Dynamics Simulation: A Case of Oligopeptide Binding Protein. Computational and Mathematical Methods In Medicine, 2018, 1-12.

- Fuhrmann, J., Rurainski, A., Lenhof, H., \& Neumann, D. (2010). A new Lamarckian genetic algorithm for flexible ligand-receptor docking. Journal of Computational Chemistry, NA-NA.

- Fujita, M. (2002). Design, synthesis, and bioactivities of novel diarylthiophenes: inhibitors of tumor necrosis factor- $\alpha$ (TNF- $\alpha$ ) production. Bioorganic \& Medicinal Chemistry, 10(10), 3113-3122.

- Gao, Q., Yang, L., \& Zhu, Y. (2010). Pharmacophore Based Drug Design Approach as a Practical Process in Drug Discovery. Current Computer Aided-Drug Design, 6(1), 37-49. 
- Gasteiger, J. (2005). Chemoinformatics: a new field with a long tradition. Analytical and Bioanalytical Chemistry, 384(1), pp.57-64.

- Ghose, A., Viswanadhan, V., \& Wendoloski, J. (1999). A Knowledge-Based Approach in Designing Combinatorial or Medicinal Chemistry Libraries for Drug Discovery. 1. A Qualitative and Quantitative Characterization of Known Drug Databases. Journal of Combinatorial Chemistry, 1(1), 55-68.

- GRANT, J., GALlARDO, M., \& PICKUP, B. (1996). A fast method of molecular shape comparison: A simple application of a Gaussian description of molecular shape. Journal of Computational Chemistry, 17(14), 1653-1666.

- Guirado, A., López Sánchez, J., Ruiz-Alcaraz, A., García-Peñarrubia, P., Bautista, D., \& Gálvez, J. (2013). First synthesis and biological evaluation of 4-amino-2-aryl-6,9dichlorobenzo[g]pteridines as inhibitors of TNF- $\alpha$ and IL-6. European Journal of Medicinal Chemistry, 66, 269-275.

- Hanumanthappa, P., K. Teli, M., \& G. Krishnamurthy, R. (2012). Generation of Pharmacophore and Atom Based 3D-QSAR Model of Novel Isoquinolin-1-one and Quinazolin-4-one-type Inhibitors of TNFa. Medicinal Chemistry, 8(3), 436-451.

- He, M. (2005). Small-Molecule Inhibition of TNF-. Science, 310(5750), 1022-1025.

- Hess, B., Bekker, H., Berendsen, H., \& Fraaije, J. (1997). LINCS: A linear constraint solver for molecular simulations. Journal of Computational Chemistry, 18(12), 1463-1472.

- Hochberg, M., Lebwohl, M., Plevy, S., Hobbs, K., \& Yocum, D. (2005). The Benefit/Risk Profile of TNF-Blocking Agents: Findings of a Consensus Panel. Seminars In Arthritis And Rheumatism, 34(6), 819-836.

- Idriss, H., \& Naismith, J. (2000). TNF? and the TNF receptor superfamily: Structurefunction relationship(s). Microscopy Research and Technique, 50(3), 184-195.

- Irwin, J. J. \& Shoichet, B. K. 2005. ZINC a free database of commercially available compounds for virtual screening. Journal of Chemical Informatics and Modeling, 45, 177182. 
- Jacobi, A., Mahler, V., Schuler, G., \& Hertl, M. (2006). Treatment of inflammatory dermatoses by tumor necrosis factor antagonists. Journal of The European Academy Of Dermatology And Venereology, 20(10), 1171-1187.

- Jang, J., Yoon, H., Yoon, J., Kim, H., Lee, S., \& Kim, K. et al. (2009). Crystal Structure of the TNF- $\alpha$-Inducing Protein (Tip $\alpha$ ) from Helicobacter pylori: Insights into Its DNABinding Activity. Journal of Molecular Biology, 392(1), 191-197.

- Jana, S., \& Paliwal, J. (2007). Molecular Mechanisms of Cytochrome P450 Induction: Potential for Drug-Drug Interactions. Current Protein \& Peptide Science, 8(6), 619-628.

- Kang, T., Mao, Z., Ng, C., Wang, M., Wang, W., \& Wang, C. et al. (2016). Identification of an Iridium(III)-Based Inhibitor of Tumor Necrosis Factor- $\alpha$. Journal of Medicinal Chemistry, 59(8), 4026-4031.

- Kim, Y., Hong, Y., Joo, Y., Woo, B., Kim, S., \& Koh, H. et al. (2014). Synthesis and structure-activity relationship of cyclopentenone oximes as novel inhibitors of the production of tumor necrosis factor- $\alpha$. Bioorganic \& Medicinal Chemistry Letters, 24(13), 2807-2810.

- Laskowski, R., MacArthur, M., Moss, D., \& Thornton, J. (1993). PROCHECK: a program to check the stereochemical quality of protein structures. Journal of Applied Crystallography, 26(2), 283-291.

- Laufersweiler, M., \& et al., e. (2004). The Development of Novel Inhibitors of Tumor Necrosis Factor- $\alpha$ (TNF- $\alpha$ ) Production Based on Substituted [5,5]-Bicyclic Pyrazolones. Cheminform, 35(49).

- Leung, C., Zhong, H., Yang, H., Cheng, Z., Chan, D., \& Ma, V. et al. (2012). A Metalbased Inhibitor of Tumor Necrosis Factor- $\alpha$. Angewandte Chemie International Edition, 51(36), 9010-9014.

- Leung, C., Chan, D., Kwan, M., Cheng, Z., Wong, C., \& Zhu, G. et al. (2011). StructureBased Repurposing of FDA-Approved Drugs as TNF- $\alpha$ Inhibitors. Chemmedchem, 6(5), 765-768.

- Liang, S., Dai, J., Hou, S., Su, L., Zhang, D., \& Guo, H. et al. (2013). Structural Basis for Treating Tumor Necrosis Factor $\alpha(\mathrm{TNF}-\alpha)$-associated Diseases with the Therapeutic Antibody Infliximab. Journal Of Biological Chemistry, 288(19), 13799-13807. 
- Li, Z., Wan, H., Shi, Y. and Ouyang, P. (2004). Personal Experience with Four Kinds of Chemical Structure Drawing Software: Review on ChemDraw, ChemWindow, ISIS/Draw, and ChemSketch. Journal of Chemical Information and Computer Sciences, 44(5), pp.1886-1890.

- Lin, J., \& Yamazaki, M. (2003). Role of P-Glycoprotein in Pharmacokinetics. Clinical Pharmacokinetics, 42(1), 59-98.

- Lis, K., Kuzawińska, O., \& Bałkowiec-Iskra, E. (2014). State of the art paper Tumor necrosis factor inhibitors - state of knowledge. Archives of Medical Science, 6, 1175-1185.

- Lipinski, C., Lombardo, F., Dominy, B., \& Feeney, P. (2001). Experimental and computational approaches to estimate solubility and permeability in drug discovery and development settings 1PII of original article: S0169-409X(96)00423-1. The article was originally published in Advanced Drug Delivery Reviews 23 (1997) 3-25. 1. Advanced Drug Delivery Reviews, 46(1-3), 3-26.

- Lipinski, C. (2004). Lead- and drug-like compounds: the rule-of-five revolution. Drug Discovery Today: Technologies, 1(4), 337-341.

- Lobanov, M., Bogatyreva, N., \& Galzitskaya, O. (2008). The radius of gyration as an indicator of protein structure compactness. Molecular Biology, 42(4), 623-628.

- Louis S. (2007). Sybyl, version 7.3; software for molecular modeling package.

- Lüthy, R., Bowie, J., \& Eisenberg, D. (1992). Assessment of protein models with threedimensional profiles. Nature, 356(6364), 83-85.

- Ma, L., Gong, H., Zhu, H., Ji, Q., Su, P., \& Liu, P. et al. (2014). A Novel Small-molecule Tumor Necrosis Factor $\alpha$ Inhibitor Attenuates Inflammation in a Hepatitis Mouse Model. Journal Of Biological Chemistry, 289(18), 12457-12466.

- Malde, A., Zuo, L., Breeze, M., Stroet, M., Poger, D., \& Nair, P. et al. (2011). An Automated Force Field Topology Builder (ATB) and Repository: Version 1.0. Journal of Chemical Theory And Computation, 7(12), 4026-4037.

- Mancini, F., Toro, C., Mabilia, M., Giannangeli, M., Pinza, M., \& Milanese, C. (1999). Inhibition of tumor necrosis factor- $\alpha(\mathrm{TNF}-\alpha) / \mathrm{TNF}-\alpha$ receptor binding by structural 
analogs of suramin§§Abbreviations: TNF- $\alpha$, tumor necrosis factor- $\alpha$; and $\mathrm{MC} / \mathrm{EM}$, MonteCarlo/energy minimization. Biochemical Pharmacology, 58(5), 851-859.

- Mark, P., \& Nilsson, L. (2001). Structure and Dynamics of the TIP3P, SPC, and SPC/E Water Models at 298 K. The Journal of Physical Chemistry A, 105(43), 9954-9960.

- Maxwell, L., Zochling, J., Boonen, A., Singh, J., Veras, M., \& Tanjong Ghogomu, E. et al. (2015). TNF-alpha inhibitors for ankylosing spondylitis. Cochrane Database of Systematic Reviews, Art. No.: CD005468.(4).

- McInnes, I., Buckley, C., \& Isaacs, J. (2015). Cytokines in rheumatoid arthritis - shaping the immunological landscape. Nature Reviews Rheumatology, 12(1), 63-68.

- Mesaik, M., Jabeen, A., Halim, S., Begum, A., Khalid, A., \& Asif, M. et al. (2012). In Silico and In Vitro Immunomodulatory Studies on Compounds of Lindelofia stylosa. Chemical Biology \& Drug Design, 79(3), 290-299.

- Monaco, C., Nanchahal, J., Taylor, P., \& Feldmann, M. (2014). Anti-TNF therapy: past, present, and future. International Immunology, 27(1), 55-62.

- Morris, G., Huey, R., Lindstrom, W., Sanner, M., Belew, R., Goodsell, D. and Olson, A. (2009). AutoDock4 and AutoDockTools4: Automated docking with selective receptor flexibility. Journal of Computational Chemistry, 30(16), pp.2785-2791.

- Mouchlis, V., Melagraki, G., Mavromoustakos, T., Kollias, G. and Afantitis, A. (2012). Molecular Modeling on Pyrimidine-Urea Inhibitors of TNF- $\alpha$ Production: An Integrated Approach Using a Combination of Molecular Docking, Classification Techniques, and 3DQSAR CoMSIA. Journal of Chemical Information and Modeling, 52(3), pp.711-723.

- Mouhsine, H., Guillemain, H., Moreau, G., Fourati, N., Zerrouki, C., \& Baron, B. et al. (2017). Identification of an in vivo orally active dual-binding protein-protein interaction inhibitor targeting TNF $\alpha$ through combined in silico/in vitro/in vivo screening. Scientific Reports, 7(1).

- Muegge, I. (2003). Selection Criteria for Drug-Like Compounds. Cheminform, 34(29).

- Mysinger, M., Carchia, M., Irwin, J., \& Shoichet, B. (2012). Directory of Useful Decoys Enhanced (DUD-E): Better Ligands and Decoys for Better Benchmarking. Journal of Medicinal Chemistry, 55(14), 6582-6594. 
- Palladino, M., Bahjat, F., Theodorakis, E., \& Moldawer, L. (2003). Anti-TNF- $\alpha$ therapies: the next generation. Nature Reviews Drug Discovery, 2(9), 736-746.

- Papadakis, K., \& Targan, S. (2000). Tumor necrosis factor: Biology and therapeutic inhibitors. Gastroenterology, 119(4), 1148-1157.

- Pérez, A., Marchán, I., Svozil, D., Sponer, J., Cheatham, T., Laughton, C. and Orozco, M. (2007). Refinement of the AMBER Force Field for Nucleic Acids: Improving the Description of $\alpha / \gamma$ Conformers. Biophysical Journal, 92(11), pp.3817-3829.

- Ramachandran, G., Ramakrishnan, C., \& Sasisekharan, V. (1963). Stereochemistry of polypeptide chain configurations. Journal of Molecular Biology, 7(1), 95-99.

- Sabat, M., \& et al., e. (2006). The Development of Novel C-2, C-8, and N-9 Trisubstituted Purines as Inhibitors of TNF- $\alpha$ Production. Cheminform, 37(51).

- Sander, T., Freyss, J., von Korff, M., \& Rufener, C. (2015). DataWarrior: An Open-Source Program for Chemistry Aware Data Visualization and Analysis. Journal of Chemical Information and Modeling, 55(2), 460-473.

- Scott, W., Hünenberger, P., Tironi, I., Mark, A., Billeter, S., \& Fennen, J. et al. (1999). The GROMOS Biomolecular Simulation Program Package. The Journal of Physical Chemistry A, 103(19), 3596-3607.

- SCRGUIDE 2004. The Maybridge Screening Collection- Maybridge... Bringing Life to Drug Discovery. Fisher Scientific.

- Sedger, L., \& McDermott, M. (2014). TNF and TNF-receptors: From mediators of cell death and inflammation to therapeutic giants - past, present, and future. Cytokine \& Growth Factor Reviews, 25(4), 453-472.

- Sfikakis, P., \& Tsokos, G. (2011). Towards the next generation of anti-TNF drugs. Clinical Immunology, 141(3), 231-235.

- Shah, M., Arfan, M., Amin, H., Hussain, Z., Qadir, M., \& Iqbal Choudhary, M. et al. (2012). Synthesis of new bergenin derivatives as potent inhibitors of inflammatory mediators NO and TNF- $\alpha$. Bioorganic \& Medicinal Chemistry Letters, 22(8), 2744-2747. 
- Singh, U., \& Kollman, P. (1984). An approach to computing electrostatic charges for molecules. Journal of Computational Chemistry, 5(2), 129-145.

- Stinson, S. F., Alley, M. C., Kopp, W. C., Fiebig, H. H., Mullendore, L. A., Pittman, A. F., Kenney, S., Keller, J. \& Boyd, M. R. 1992. Morphological and immunocytochemical characteristics of human tumor cell lines for use in a disease-oriented anticancer drug screen. Anticancer Research, 12, 1035-1053.

- Sticherling, M. (2016). Psoriasis and autoimmunity. Autoimmunity Reviews, 15(12), 11671170.

- Tang, P., Hung, M., \& Klostergaard, J. (1996). Human pro-Tumor Necrosis Factor Is a Homotrimer. Biochemistry, 35(25), 8216-8225.

- Triballeau, N., Acher, F., Brabet, I., Pin, J., \& Bertrand, H. (2005). Virtual Screening Workflow Development Guided by the "Receiver Operating Characteristic" Curve Approach. Application to High-Throughput Docking on Metabotropic Glutamate Receptor Subtype 4. Journal of Medicinal Chemistry, 48(7), 2534-2547.

- Truchon, J., \& Bayly, C. (2007). Evaluating Virtual Screening Methods: Good and Bad Metrics for the "Early Recognition" Problem. Journal of Chemical Information And Modeling, 47(2), 488-508.

- Version, O. 2006. 2.1, OpenEye Scientific Software. 2.1 ed.

- Veber DF, Johnson SR, Cheng HY, Smith BR, Ward KW, et al. (2002) Molecular properties that influence the oral bioavailability of drug candidates. J Med Chem 45: 26152623.

- Vilar, S., Cozza, G., \& Moro, S. (2008). Medicinal Chemistry and the Molecular Operating Environment (MOE): Application of QSAR and Molecular Docking to Drug Discovery. Current Topics in Medicinal Chemistry, 8(18), 1555-1572

- Wajant, H., Pfizenmaier, K., \& Scheurich, P. (2003). Tumor necrosis factor signaling. Cell Death \& Differentiation, 10(1), 45-65.

- Willett, P., Barnard, J., \& Downs, G. (1998). Chemical Similarity Searching. Journal of Chemical Information and Computer Sciences, 38(6), 983-996.

- Wolber, G., \& Langer, T. (2005). LigandScout: 3-D Pharmacophores Derived from Protein-Bound Lingands and Their Use as Virtual Screening Filters. Cheminform, 36(16). 
- Zaka, M., Sehgal, S. A., Shafique, S., \& Abbasi, B. H. (2017). Comparative in silico analyses of Cannabis sativa, Prunella vulgaris and Withania somnifera compounds elucidating the medicinal properties against rheumatoid arthritis. J Mol Graph Model, 74, 296-304. 